\title{
Lagrangian Measurement of Steep Directionally Spread Ocean Waves: Second-Order Motion of a Wave-Following Measurement Buoy
}

\author{
M. L. MCAlLisTer AND T. S. VAN DEN BREMER \\ Department of Engineering Science, University of Oxford, Oxford, United Kingdom
}

(Manuscript received 28 July 2019, in final form 23 September 2019)

\begin{abstract}
The notion that wave-following buoys provide less accurate measurements of extreme waves than their Eulerian counterparts is a perception commonly held by oceanographers and engineers (Forristall 2000, J. Phys. Oceanogr., 30, 1931-1943, https://doi.org/10.1175/1520-0485(2000)030<1931:WCDOAS>2.0.CO;2). By performing a direct comparison between the two types of measurement under laboratory conditions, we examine one of the hypotheses underlying this perception and establish whether wave measurement buoys in extreme ocean waves correctly follow steep crests and behave in a purely Lagrangian manner. We present a direct comparison between Eulerian gauge and Lagrangian buoy measurements of steep directionally spread and crossing wave groups on deep water. Our experimental measurements are compared with exact (Herbers and Janssen 2016, J. Phys. Oceanogr., 46, 1009-1021, https://doi.org/10.1175/JPO-D-15-0129.1) and new approximate expressions for Lagrangian second-order theory derived herein. We derive simple closed-form expressions for the second-order contribution to crest height representative of extreme ocean waves-namely, for a single narrowly spread wave group, two narrowly spread crossing wave groups, and a single strongly spread wave group. In the limit of large spreading or head-on crossing, Eulerian and Lagrangian measurements become equivalent. For the range of conditions that we test, we find that our buoy behaves in a Lagrangian manner, and our experimental observations compare extremely well to predictions made using second-order theory. In general, Eulerian and Lagrangian measurements of crest height are not significantly different for all degrees of directional spreading and crossing. However, second-order boundwave energy is redistributed from superharmonics in Eulerian measurements to subharmonics in Lagrangian measurement, which affects the "apparent" steepness inferred from time histories and poses a potential issue for wave buoys that measure acceleration.
\end{abstract}

\section{Introduction}

Wave-following buoys are commonly used as devices to measure free surface elevation in the oceans. This is, in part, owing to their relative ease of installation; unlike the majority of Eulerian measurement devices, buoys do not require a supporting structure. Measurements made with both buoys and Eulerian devices are used in operational oceanography and ocean engineering in various forms. Summary statistics such as significant wave height $H_{s}$ and peak period $T_{p}$ are commonly used to calibrate phase-averaged ocean forecast and hindcast models (Janssen et al. 1997; Bidlot et al. 2002) and to characterize environmental conditions across the oceans (Hasselmann et al. 1980; Pontes 1998; Mork et al. 2010; Fedele and Arena 2010; Lenee-Bluhm et al. 2011).

Corresponding author: M. L. McAllister, mark.mcallister@ eng.ox.ac.uk
Time-domain measurements are used to investigate wave dynamics and to gain insight into processes such as wave breaking and freak wave formation (Holthuijsen and Herbers 1986; Guedes Soares et al. 2004; Waseda et al. 2014; Whittaker et al. 2016). In such studies, the two types of measurement are often used interchangeably.

However, within the oceanographic and ocean engineering communities, it is generally perceived that wave buoys are less accurate, particularly when concerning the measurement of extreme waves (Allender et al. 1989; Forristall 2000; Dysthe and Müller 2008; Collins et al. 2014). Various explanations are offered for this perceived inaccuracy, such as a lack of compliance in the mooring configuration or the ability of a buoy to move horizontally causing it avoid the highest crests (Allender et al. 1989; Krogstad and Barstow 2000), the cancellation of second-order superharmonic contributions to crest height owing to their Lagrangian motion (Longuet-Higgins 1986; Rademakers 1993), and low 
sampling frequencies resulting in wave crests being missed (Forristall 2000). For completeness, we note the probability of observing extreme waves in single-point observations (Eulerian or Lagrangian) is different from spatial-temporal observations (e.g., Benetazzo et al. 2017), although we do not focus on this herein.

In principle, a floating wave-following buoy measures the Lagrangian free surface elevation $\eta_{L}\left[\mathbf{x}_{H}(t), t\right]$, which is function of time $t$ both directly and indirectly through its dependence on its time-varying horizontal position $\mathbf{x}_{H}(t)$, unlike an Eulerian measurement $\eta_{E}\left(\mathbf{x}_{H, 0}, t\right)$, which is made at a fixed location $\mathbf{x}_{H, 0}$. In the limit in which waves are of small amplitude and their behavior is approximated well by linear theory, Lagrangian and Eulerian measurements are identical. The nonlinearity associated with steep waves can cause the two types of measurement to differ.

Longuet-Higgins (1986) examined this for regular progressive waves on deep water, showing how measurements may differ owing to nonlinear effects that occur at second-order in amplitude. The horizontal motion of a Lagrangian observer serves to cancel out the sharpening of crests and broadening of troughs, observed in an Eulerian frame of reference that is a result of secondorder superharmonic wave components. This also results in an increase in the position of the mean free surface in Lagrangian measurements by approximately $a^{2} k / 2$, when compared with an Eulerian measurement, where $a$ is the amplitude of a wave, $k=2 \pi / \lambda$ is its wavenumber, and $\lambda$ is its wavelength (Longuet-Higgins 1986). Additionally, the Stokes drift velocity $u_{S}$ causes a Doppler shift of the wave speed $c$ (in the Eulerian reference frame), and the measured wave period in the Lagrangian reference frame $T_{L}$ increases relative to the period in the Eulerian reference frame $T_{E}$ (LonguetHiggins 1986):

$$
\frac{T_{L}-T_{E}}{T_{E}}=\frac{u_{S}}{c-u_{S}}
$$

which has recently been studied experimentally by Grue and Kolaas (2019) for finite depth.

Following on from Srokosz and Longuet-Higgins (1986), Herbers and Janssen (2016, hereafter HJ16) investigated how second-order effects apply to irregular waves on finite depth by extending existing Eulerian multicomponent second-order theory (see Hasselmann 1962; Longuet-Higgins and Stewart 1962; Sharma and Dean 1981; Dalzell 1999) to a Lagrangian reference frame through the inclusion of an additional term to account for horizontal motion of surface-following particle. HJ16 illustrated that for irregular waves on deep water, much like regular waves, the position of the "mean" or "wave averaged" free surface (subharmonic bound-wave components at second order) is shifted up. Where a "setdown" (negative depression) is observed in an Eulerian measurement of a wave group, a "setup" (positive hump) is observed in the corresponding Lagrangian measurement. Similarly, as for a regular wave, superharmonic components disappear in a Lagrangian measurement. HJ16 note that for waves on shallow water, the Eulerian subharmonic secondorder bound wave components approach resonance and are strongly amplified, dominating over the Lagrangian components, which are not amplified by water depth in the same way. Thus, Lagrangian measurements in shallow water are approximately equal to their Eulerian equivalents.

A number of studies have considered the contribution that second-order nonlinearity makes to the crest height of extreme waves in an Eulerian frame of reference, finding that the local directional conditions have a strong effect. For steep wave groups in unidirectional and weakly spread conditions, bound second-order subharmonic or "difference" waves form a depression in the wave-averaged surface elevation often referred to as a setdown (Longuet-Higgins and Stewart 1962). In the case of crossing and strongly directionally spread wave systems, the setdown of subharmonic bound waves underneath a wave group can turn into a setup, enhancing the maximum crest amplitude, as theoretically predicted (Okihiro et al. 1992; Herbers et al. 1994; Toffoli et al. 2006; Christou et al. 2009) based on second-order interaction kernels (Hasselmann 1962; Sharma and Dean 1981; Dalzell 1999; Forristall 2000), observed in field data (Santo et al. 2013; Walker et al. 2004; Toffoli et al. 2007) and recently in detailed laboratory experiments (McAllister et al. 2018).

Wave measurement buoys are positively buoyant and float partially submerged on the water surface. The Datawell BV Waverider buoy and other simple spherical buoys operate approximately half submerged and typically have densities in the range 0.55 $0.66 \rho_{w}$, where $\rho_{w}$ is the density of seawater. Because these buoys are neither neutrally buoyant nor completely submerged, they will not necessarily behave in a purely Lagrangian manner. In different physical contexts, enhanced Stokes drift has been predicted due to the particle's inertia for very small submerged particles (Santamaria et al. 2013), due to the particle sliding down the slope for floating plates (Meylan et al. 2015) and due to wave breaking (Pizzo et al. 2019). Additionally, wave measurement buoys are often moored (to the seabed); the force provided by a mooring system may also cause buoy motion to deviate from purely Lagrangian. 
In practice, measurement buoys can be deployed without a mooring, but are most commonly moored. Measurement buoy moorings are designed to have minimal effect on their motion; the resulting designs are complex and vary significantly depending on the depth of water they are installed in. Their general design comprises a slack buoyant inflexible section connected to the seabed, which allows for a large range of lateral motion. From this, a section composed of a flexible material then connects to the buoy itself. The flexibility of this second section reduces its effect on the heave motion of the buoy.

Short of side-by-side comparison, it is difficult to establish to what extent a buoy will behave in a true Lagrangian manner in the ocean and how its measurements will compare to measurements of the Eulerian free surface elevation at the same location. Various studies have made comparisons of in situ measurements between various buoy designs (Allender et al. 1989; O'Reilly et al. 1996; Ashton and Johanning 2015; Raghukumar et al. 2019) and with other types of sensors (O'Reilly et al. 1996; Forristall et al. 2004; Herbers et al. 2012). Liu et al. (2015) carried out experiments to investigate the performance of slack-moored disc-type wave buoys, which they compared with nearby gauge measurements, finding good agreement when considering the spectral parameters significant wave height and peak period.

To the authors' knowledge, there are no studies that make a direct comparison between Eulerian and Lagrangian wave-following measurement devices and that focus on second-order aspects of the motion. In this paper, we perform a series of laboratory experiments with a scaled model buoy to make exactly this comparison and thus provide experimental validation of the Lagrangian second-order theory of HJ16. Using the multiple-scales method, we derive new narrow-banded approximations to the multichromatic theory of HJ16 for three cases: a single weakly directionally spread wave group, two crossing weakly directionally spread wave groups, and a single strongly directionally spread wave group. The resulting expressions are simple and allow a direct appreciation of the magnitude of the secondorder sub- and superharmonic bound waves, as well as their physical origins in different cases.

In carrying out our experiments, we set out to (partially) answer the question whether wave-following buoys (without mooring) behave in a purely Lagrangian manner. In particular, we consider the behavior of scaled spherical model buoys in steep directionally spread and crossing wave groups on deep water. We focus on wave groups because they provide a good approximation of the shape of extreme waves in the ocean (Lindgren 1970;
Boccotti 1983; Phillips et al. 1993; Santo et al. 2013; Gemmrich and Thomson 2017).

This paper is laid out as follows. First, in section 2, we review and compare existing Eulerian and Lagrangian second-order theory, and present our novel approximate second-order expressions derived for waves groups. We then give details of our experimental method in section 3, and present our results in section 4 . We discuss the generality of our findings and draw conclusions in section 5 .

\section{Second-order theory}

In this section, we introduce existing Eulerian and Lagrangian multichromatic second-order theory (section $2 b$ ). We then present our new narrow-banded approximations, derived using the multiple-scales method, for three cases: a single weakly directionally spread wave group (WSG) (section 2c), two crossing wave groups (CWGs) (section $2 \mathrm{~d}$ ), and a single strongly directionally spread wave group (SSG) (section 2e). We also evaluate the Stokes drift velocity and the resulting change in the Lagrangian frequency for each of these three cases. Motivated by ocean applications, we focus on cases where the water is deep relative to the wavelength of the carrier wave (i.e., $k_{0} d \gg 1$, with $k_{0}=2 \pi / \lambda_{0}$ being the carrier wavenumber and $d$ being depth).

\section{a. Governing equations}

We assume a three-dimensional body of water of depth $d$ and indefinite lateral extent with a coordinate system $(x, y, z)$, where $x$ and $y$ denote the horizontal coordinates and $z$ is the vertical coordinate measured from the undisturbed water level upward. Inviscid, incompressible and irrotational flow is assumed, and, as a result, the velocity vector can be defined as the gradient of the velocity potential, $\mathbf{u}=\nabla \phi$, and the governing equation within the domain of the fluid is Laplace,

$$
\nabla^{2} \phi=0 \text { for }-d \leq z \leq \eta(x, y, t),
$$

where $\eta(x, y, t)$ denotes the free surface. The kinematic and dynamic free surface boundary conditions at $z=$ $\eta(x, y, t)$ are respectively

$$
w-\frac{\partial \eta}{\partial t}-u \frac{\partial \eta}{\partial x}-v \frac{\partial \eta}{\partial y}=0 \quad \text { and } \quad g \eta+\frac{\partial \phi}{\partial t}+\frac{1}{2}|\nabla \phi|^{2}=0
$$

where gravity $g$ acts in the negative $z$ direction and $|\nabla \phi|^{2}=u^{2}+v^{2}+w^{2}$. There is a no-flow bottom boundary condition requiring that $\partial \phi / \partial z=0$ at $z=-d$. By retaining terms up to quadratic in the amplitude of 
the waves, the two free surface boundary conditions in Eq. (3) can be combined into two forcing equations for the second-order potential and free surface, respectively (e.g., Longuet-Higgins and Stewart 1964; McAllister et al. 2018):

$$
\begin{aligned}
\left.\left(\frac{\partial}{\partial z}+\frac{1}{g} \frac{\partial^{2}}{\partial t^{2}}\right) \phi_{E}^{(2)}\right|_{z=0}= & \left.\nabla_{H} \cdot\left[\mathbf{u}_{H}^{(1)} \eta^{(1)}\right]\right|_{z=0} \\
& -\left.\frac{1}{g} \frac{\partial}{\partial t}\left[\frac{\partial^{2} \phi^{(1)}}{\partial z \partial t} \eta^{(1)}+\frac{1}{2}|\nabla \phi|^{2}\right]\right|_{z=0}
\end{aligned}
$$

and

$$
\eta_{E}^{(2)}=\left.\frac{-1}{g}\left\{\frac{\partial \phi_{E}^{(2)}}{\partial t}+\left[\frac{\partial^{2} \phi^{(1)}}{\partial z \partial t} \eta^{(1)}+\frac{1}{2}|\nabla \phi|^{2}\right]\right\}\right|_{z=0},
$$

where the superscripts denote the order in amplitude and we use (superfluous) subscripts $E$ to denote Eulerian to allow distinction with Lagrangian $(L)$ to be made below (note that we only introduce these subscripts for second-order quantities). For simplicity, we will refer to fields or quantities recorded by a Lagrangian observer as Lagrangian-for example, Lagrangian free surface $\eta_{L}$ and Lagrangian frequency $\omega_{L}$. The subscript $H$ denotes horizontal components only, so that $\mathbf{u}_{H}=(u, v, 0)$.

\section{b. Multicomponent second-order theory (review)}

Assuming independence between the directional $\Omega(\theta)$ and amplitude $\hat{\eta}(\omega)$ distributions, the linear free surface can be given by a summation over $N_{k}$ discrete components in $N_{\theta}$ directions:

$$
\eta^{(1)}(\mathbf{x}, t)=\sum_{n=1}^{N_{k}} \sum_{i=1}^{N_{\theta}} \Omega\left(\theta_{i}\right) \hat{\eta}_{n} \cos \left(\varphi_{n, i}\right) \delta k \delta \theta,
$$

where $\varphi_{n, i}=\mathbf{k}_{n, i} \cdot \mathbf{x}_{H}-\omega_{n} t+\mu_{n, i}$ and the wavenumber vector $\mathbf{k}_{n, i}=k_{n}\left[\cos \left(\theta_{i}\right), \sin \left(\theta_{i}\right)\right]$ has magnitude $k_{n}$, with $\theta$ being measured anticlockwise from the positive $x$ axis. Frequencies and wavenumbers are related by the linear dispersion relationship

$$
\omega_{n}=\left[g\left|\mathbf{k}_{n}\right| \tanh \left(\left|\mathbf{k}_{n}\right| d\right)\right]^{1 / 2} .
$$

Similarly, the linear potential is

$$
\phi^{(1)}(\mathbf{x}, t)=\sum_{n=1}^{N_{k}} \sum_{i=1}^{N_{\theta}} \frac{\omega_{n}}{\left|\mathbf{k}_{n}\right|} \Omega\left(\theta_{i}\right) \hat{\eta}_{n} e^{\left|\mathbf{k}_{n}\right| z} \sin \left(\varphi_{n, i}\right) \delta k \delta \theta .
$$

Evaluating Eqs. (4) and (5) using these linear solutions results in the following expression for second-order free surface elevation,

$$
\begin{aligned}
\eta_{ \pm}^{(2)}(\mathbf{x}, t)= & \sum_{n=1}^{N_{k}} \sum_{m=1}^{N_{k}} \sum_{i=1}^{N_{\theta}} \sum_{j=1}^{N_{\theta}} \hat{\eta}_{n} \hat{\eta}_{m} \Omega\left(\theta_{i}\right) \Omega\left(\theta_{j}\right) \\
& \times B_{m, n, i, j}^{ \pm} \cos \left(\varphi_{n, i} \pm \varphi_{m, j}\right)(\delta k)^{2}(\delta \theta)^{2}
\end{aligned}
$$

where the subscript \pm denotes bound waves that occur as the sum $(+)$ or difference $(-)$ of the frequencies of their interacting parent waves and $B^{ \pm}$represent the respective second-order interaction kernels. The structure of these second-order interaction kernels may be defined to account for Eulerian $B_{E}^{ \pm}$(e.g., Dalzell 1999) or Lagrangian $B_{L}^{ \pm}$(HJ16) free surface motion.

Although expressions for the "frequency difference" terms in a multidirectional sea can be distilled from Hasselmann (1962) (cf. Okihiro et al. 1992), Sharma and Dean (1981), Dalzell (1999), and Forristall (2000) are commonly credited for extending the work of LonguetHiggins and Stewart (1962) to finite-depth directional seas, allowing for interactions between-parent wave components of different frequencies and traveling in different directions [see Pellet et al. (2017) for a recent rederivation that also includes pressure].

Restricting our attention to terms up to second-order in amplitude, the Eulerian and the Lagrangian free surface are related by

$$
\eta_{L}^{(2)}=\eta_{E}^{(2)}+\Delta \eta_{L}^{(2)} \quad \text { with } \quad \Delta \eta_{L}^{(2)}=\Delta \mathbf{x}_{H}^{(1)} \cdot \nabla_{H} \eta^{(1)},
$$

where $\Delta \mathbf{x}_{H}^{(1)}$ is the linear horizontal motion (i.e., $\left.\partial \Delta \mathbf{x}_{H}^{(1)} / \partial t=\mathbf{u}_{H}^{(1)}\right)$. Resulting expressions for the secondorder interaction kernels for a Lagrangian observer were derived in a unidirectional framework by Srokosz and Longuet-Higgins (1986) and Prevosto et al. (2000) and, more recently, in fully directional seas by HJ16.

Distinguishing super- and subharmonic (or mean) terms, we will now consider the Eulerian and Lagrangian surface elevation when the spectrum is narrow banded for three cases: a single WSG (section 2c), two CWGs (section 2d), and a single SSG (section 2e). Our intention here is to develop an understanding of the behavior at second-order that cannot be directly inferred from the complicated kernels. In doing so, we derive simple expressions for the second-order Eulerian and Lagrangian free surface elevations and frequencies. To do so, we thus focus on narrow-banded spectra and derive results using the method of multiple scales, considering only leading-order terms.

In each case, we implement the following nondimensionalization: $\tilde{\eta}^{(2)} \equiv \eta^{(2)} /\left(k_{0} a_{0}^{2} / 2\right)$, where the notation $\tilde{\eta}^{(2)}$ also indicates that our expressions are evaluated at the point of focus $(x=0, y=0)$, only the dependence on the fast time scale of the linear waves is maintained, and $\left.a_{0} \equiv \mid A(t=0), y=0, t=0\right) \mid$ is the 
focused linear amplitude of the surface elevation envelope $A$. We choose this point because the second-order surface elevations reach their extrema here. Results are summarized in Table 1 and are compared with exact multicomponent second-order theory in section $2 \mathrm{f}$.

\section{c. A single weakly spread wave group}

If we consider a WSG as a carrier wave with frequency $\omega_{0}$ and wavenumber $k_{0}$, which is slowly modulated by the envelope $A(X, Y)$, we may express the linear free surface elevation and potential respectively as

$$
\begin{aligned}
\eta^{(1)} & =A(X, Y) \cos \left(\varphi_{0}\right) \quad \text { and } \\
\phi^{(1)} & =\frac{\omega_{0}}{k_{0}} A(X, Y) e^{k_{0} z} \sin \left(\varphi_{0}\right),
\end{aligned}
$$

with $\varphi_{0}=k_{0} x-\omega_{0} t+\mu_{0}$. We have assumed that the linear waves propagate on deep water $\left(k_{0} d \gg 1\right)$ so that the linear dispersion relation becomes $\omega_{0}^{2}=g k_{0}$. The water depth is not truly infinite. In our experiments, the water depth is shallow to intermediate with respect to our wave group $\left(d / \sigma_{x} \ll 1\right.$ or $d / \sigma_{x}=O(1)$, respectively). To leading order in the multiple-scales parameter $\varepsilon\left(\varepsilon_{x} \equiv 1 /\left(k_{0} \sigma_{x}\right)=O(\varepsilon)\right.$ and $\varepsilon_{y} \equiv 1 /\left(k_{0} \sigma_{x}\right)=$ $O(\varepsilon)$, with $\sigma_{x}$ and $\sigma_{y}$ denoting the characteristic spatial scales of the group in its direction of propagation and the direction orthogonal to it, respectively), the group is a function of the slow variables: $X \equiv \varepsilon_{x}\left(x-c_{g, 0} t\right)$ and $Y \equiv \varepsilon_{y} y$, where $c_{g, 0}=d \omega_{0} / d k_{0}=\omega_{0} /\left(2 k_{0}\right)$ is the group velocity.

\section{1) EULERIAN}

From Eq. (5), it is clear that there are two contributions to the Eulerian second-order surface $\eta_{E}^{(2)}$ : from the (time derivative of the) second-order potential $\phi_{E}^{(2)}$ and from a combination of first-order terms,

$$
\eta_{E, \mathrm{LF}}^{(2)} \equiv \frac{-1}{g}\left(\left.\frac{\partial^{2} \phi^{(1)}}{\partial z \partial t}\right|_{z=0} \eta^{(1)}+\left.\frac{1}{2}|\nabla \phi|^{2}\right|_{z=0}\right),
$$

which we term the linear forcing term $\eta_{E, \mathrm{LF}}^{(2)}$. Its superharmonic component can be obtained from substituting Eq. (10) into Eq. (11):

$$
\eta_{E, \mathrm{LF},+}^{(2)}=\frac{k_{0} A(X, Y)^{2}}{2} \cos \left(2 \varphi_{0}\right)
$$

and its subharmonic component is zero in deep water $\left(k_{0} d \gg 1\right)$ unless waves cross [hence this subharmonic component was termed the crossing wave term in McAllister et al. (2018); i.e., $\eta_{E, \mathrm{CW}} \equiv \eta_{E, \mathrm{LF},-}^{(2)}$; see section $2 \mathrm{~d}]$. We can thus ignore it in the case of a single weakly spread wave group. Substituting Eq. (10) into

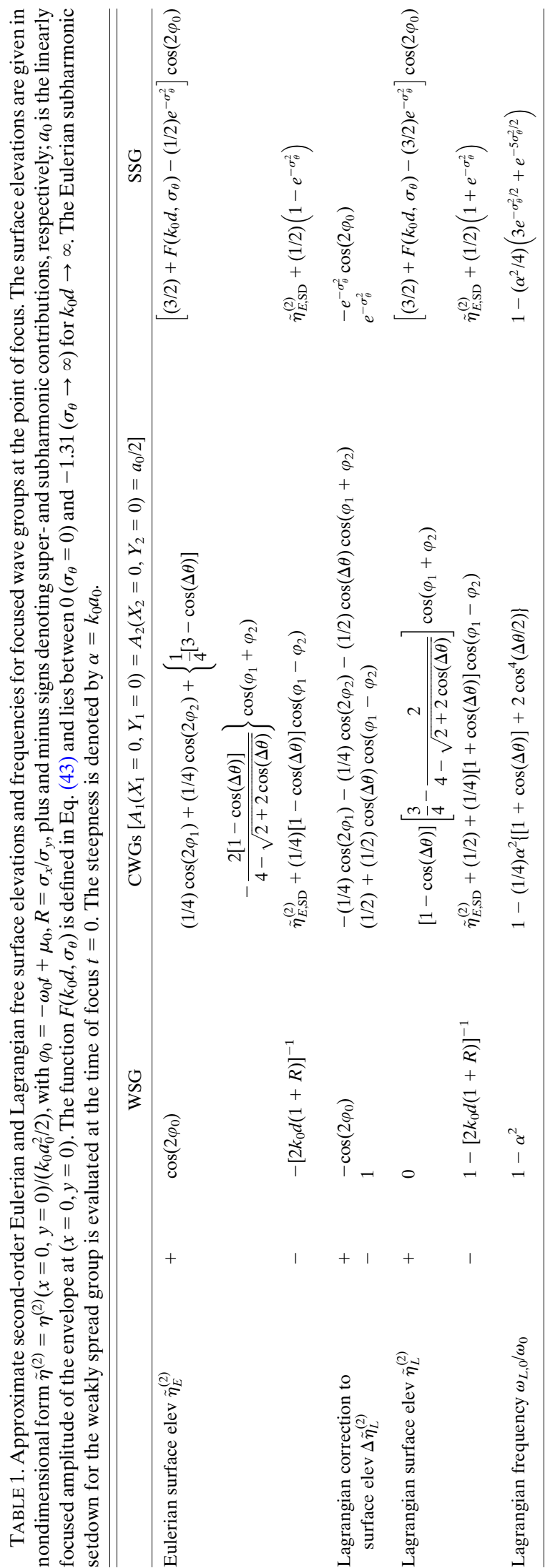


Eq. (4) and solving for $\phi_{E}^{(2)}$ yields a leading-order expression for the Eulerian second-order potential,

$$
\begin{aligned}
\phi_{E}^{(2)}= & \frac{l \omega_{0}}{8 \pi^{2}} \int_{-\infty}^{\infty} \int_{-\infty}^{\infty} \frac{\kappa \cosh \left[\sqrt{\kappa^{2}+\lambda^{2}}(z+d)\right]}{\sqrt{\kappa^{2}+\lambda^{2}} \sinh \left(\sqrt{\kappa^{2}+\lambda^{2}} d\right)} \\
& \times \widehat{A^{2}} \exp [\imath(\kappa \tilde{x}+\lambda \tilde{y})] d \kappa d \lambda,
\end{aligned}
$$

where $\tilde{x} \equiv x-c_{g, 0} t, \tilde{y} \equiv y$, and $\widehat{A^{2}}$ denotes the Fourier transform of the square of the packet. As is well known, the potential does not have a superharmonic component $\left(\phi_{E}^{(2)}=\phi_{E,-}^{(2)}\right.$; e.g., Stokes 1847). Equation (13) can then be substituted into Eq. (5) and combined with Eq. (12) to give the Eulerian free surface elevation:

$$
\begin{aligned}
\eta_{E}^{(2)}= & \frac{-1}{16 \pi^{2}} \int_{-\infty}^{\infty} \int_{-\infty}^{\infty} \frac{\kappa^{2} \widehat{A^{2}} \exp [\imath(\kappa \tilde{x}+\lambda \tilde{y})]}{\sqrt{\kappa^{2}+\lambda^{2}} \tanh \left(\sqrt{\kappa^{2}+\lambda^{2}} d\right)} d \kappa d \lambda \\
& +\frac{k_{0} A(X, Y)^{2}}{2} \cos \left(2 \varphi_{0}\right) .
\end{aligned}
$$

If we further assume that depth is shallow with respect to the group scale $\left(d / \sigma_{x} \ll 1\right)$, the double integral in Eq. (14) can be explicitly evaluated at $X=0, Y=0$ to give in nondimensional form [i.e., $\tilde{\eta}^{(2)} \equiv \eta^{(2)}(X=0$, $\left.Y=0) /\left(k_{0} a_{0}^{2} / 2\right)\right]$ (McAllister et al. 2018)

$$
\tilde{\eta}_{E}^{(2)}=-\frac{1}{2 k_{0} d} \frac{1}{1+R}+\cos \left(2 \varphi_{0}\right),
$$

where $R=\sigma_{x} / \sigma_{y}$ is the aspect ratio of the group and a measure of directional spreading, with $R \rightarrow 0$ corresponding to unidirectional waves.

Equation (15) gives the familiar result for the secondorder bound waves of a weakly directionally spread wave group. The slowly varying part represents a setdown of the free surface, which diminishes as the amount of directional spreading $R$ increases, noting that Eq. (15) is only valid for weak directional spreading. The rapidly varying superharmonics are unaffected by weak directional spreading and take the same form as for a unidirectional monochromatic wave. In fact, without loss of generality, the dependence of the superharmonic term on the rapidly varying $2 \varphi_{0}$ with $\varphi_{0}=k_{0} x-\omega_{0} t+\mu_{0}$ and on the slowly varying envelope $A(X, Y)$ can be maintained. This is not true for the slowly varying mean term representing a setdown, which can only be explicitly evaluated at the point and time of focus $(X=0, Y=0)$ (McAllister et al. 2018).

\section{2) LAGRANGIAN}

From Eq. (10), we can readily obtain the leading-order Lagrangian correction term to the second-order free surface elevation $\Delta \eta_{L}^{(2)}$, as defined in Eq. (9), which accounts for the horizontal motion of a Lagrangian observer:

$$
\Delta \eta_{L}^{(2)}=\frac{k_{0} A(X, Y)^{2}}{2}\left[1-\cos \left(2 \varphi_{0}\right)\right]
$$

which comprises a mean setup term and a superharmonic term that is out of phase with its Eulerian superharmonic counterpart in Eq. (14). Combining this with $\eta_{E}^{(2)}$ in Eq. (15), we obtain in nondimensional form

$$
\tilde{\eta}_{L}^{(2)}=1-\frac{1}{2 k_{0} d} \frac{1}{1+R} .
$$

For a single weakly spread wave group, the Lagrangian and the Eulerian superharmonic terms exactly cancel each other out, as is well known (Longuet-Higgins 1986), leaving only mean terms, as predicted in HJ16. For a Lagrangian observer, these mean terms take the form of a setup, which is only weakly diminished by the effect of the Eulerian setdown. The Lagrangian correction term $\Delta \eta_{L}^{(2)}$ does not depend on depth $d$, whereas the Eulerian term depends inversely on depth $d$ (and is small, noting that $k_{0} d \gg 1$ here).

\section{3) STOKES DRIFT VELOCITY AND LAGRANGIAN FREQUENCY}

For a Lagrangian measurement device, the measured linear wave period is increased by a Doppler shift induced by Stokes drift (Longuet-Higgins 1986), which is generally given by

$$
\mathbf{u}_{S} \equiv \overline{\Delta \mathbf{x}^{(1)} \cdot \nabla \mathbf{u}^{(1)}},
$$

which is the product of the linear displacement vector $\Delta \mathbf{x}^{(1)}$ and the gradient of the linear velocity. For a single weakly spread wave group on deep water, the Stokes drift [Eq. (18)] only has a leading-order term in the $x$ direction (the direction of propagation of the carrier wave):

$$
u_{S}=k_{0} \omega_{0} A^{2} .
$$

Using the Doppler shift $\omega_{L, 0}=k_{0}\left(c_{p, 0}-u_{S}\right)$ with $c_{p, 0}=$ $\omega_{0} / k_{0}$ being the phase velocity, we obtain the Lagrangian frequency

$$
\omega_{L, 0}=\omega_{0}\left(1-k_{0}^{2} A^{2}\right),
$$

which is equivalent to the result by Longuet-Higgins (1986) for a unidirectional monochromatic wave [except for slow dependence of the envelope and an apparently superfluous $1 / 2$ in Eq. (2.4) of Longuet-Higgins (1986)], indicating that the presence of a group or (weak) directional spreading does not play a leading-order role.

\section{d. Two crossing wave groups}

If we now consider two CWGs, with the first group traveling in the $x$ direction $\left(\theta_{p}=0\right)$ and the second 
traveling in the direction $\theta_{p}=\Delta \theta$, the linear free surface elevation and potential respectively are

$$
\begin{aligned}
\eta^{(1)}= & A_{1}\left(X_{1}, Y_{1}\right) \cos \left(\varphi_{1}\right)+A_{2}\left(X_{2}, Y_{2}\right) \cos \left(\varphi_{2}\right) \\
\phi^{(1)}= & \frac{\omega_{0}}{k_{0}} A_{1}\left(X_{1}, Y_{1}\right) e^{k_{0} z} \sin \left(\varphi_{1}\right) \\
& +\frac{\omega_{0}}{k_{0}} A_{2}\left(X_{2}, Y_{2}\right) e^{k_{0} z} \sin \left(\varphi_{2}\right)
\end{aligned}
$$

with phases $\varphi_{1}=k_{0} x-\omega_{0} t+\mu_{1}$ and $\varphi_{2}=k_{0} x \cos (\Delta \theta)+$ $k_{0} y \sin (\Delta \theta)-\omega_{0} t+\mu_{2}$. Both groups have identical frequencies $\omega_{0}$ and wavenumber magnitudes $k_{0}$, satisfying the deep-water linear dispersion relationship $\omega_{0}^{2}=g k_{0}$. Group 1 is a function of the slow scales $X_{1}=$ $\varepsilon_{x}\left(x-c_{g, 0} t\right)$ and $Y_{1}=\varepsilon_{y} y$ and group 2 is a function of $X_{2}=\varepsilon_{x}\left[x \cos (\Delta \theta)+y \sin (\Delta \theta)-c_{g, 0} t\right]$ and $Y_{1}=$ $\varepsilon_{y}[-x \sin (\Delta \theta)+y \cos (\Delta \theta)]$ so that $X_{1}$ and $X_{2}$ are in the directions of propagation of their respective groups. For simplicity, we will consider the case $A_{1}\left(X_{1}=0\right.$, $\left.Y_{1}=0\right)=A_{2}\left(X_{2}=0, Y_{2}=0\right)=a_{0} / 2$, so that the linear amplitude at the point and time of focus $\eta^{(1)}(x=0$, $y=0, t=0)=a_{0}$.

\section{1) EULERIAN}

At the subharmonic level, the second-order surface is again made up from an Eulerian setdown $\eta_{E, \mathrm{SD}}^{(2)} \equiv-(1 / g) \partial \phi_{E,-}^{(2)} / \partial t$ and a cross-wave term $\eta_{E, \mathrm{CW}}^{(2)} \equiv$ $\eta_{E, \mathrm{LF},-}^{(2)}$ [Eq. (11)]. Both have been evaluated by McAllister et al. (2018). As for the case of the weakly spread group, the magnitude of the setdown depends inversely on depth $k_{0} d$ and is generally small in deep water. The expression for the setdown is only marginally less cumbersome than the multichromatic secondorder theory reviewed in section $2 \mathrm{~b}$ and we thus do not evaluate it here. The cross-wave term gives (McAllister et al. 2018)

$$
\eta_{E, \mathrm{CW}}^{(2)}=\frac{1}{2}[1-\cos (\Delta \theta)] k_{0} A_{1} A_{2} \cos \left(\varphi_{1}-\varphi_{2}\right),
$$

where $\varphi_{1}-\varphi_{2}=x[1-\cos (\Delta \theta)]-y \sin (\Delta \theta)+\mu_{1}-\mu_{2}$. Although the setdown is always slowly varying in both time and space, the crossing-wave contribution Eq. (23) responsible for a setup is slowly varying in time, but rapidly varying in space. A partial standing wave pattern forms with lines of constant phase at an angle $\Delta \theta / 2$ to the $x$ axis-namely, in line with the bisection of the paths of travel of the two groups. Nondimensionally, we thus have for the subharmonic part of the Eulerian second-order free surface elevation at the crossing point [i.e., $\tilde{\eta}^{(2)} \equiv \eta^{(2)}(x=0, y=0) /\left(k_{0} a_{0}^{2} / 2\right)$ with $A_{1}\left(X_{1}=0\right.$, $\left.\left.Y_{1}=0\right)=A_{2}\left(X_{2}=0, Y_{2}=0\right)=a_{0} / 2\right]$,

$$
\tilde{\eta}_{E,-}^{(2)}=\tilde{\eta}_{E, \mathrm{SD}}^{(2)}+\frac{1}{4}[1-\cos (\Delta \theta)] \cos \left(\mu_{1}-\mu_{2}\right) .
$$

At the superharmonic level, terms arise from both the second-order potential $\phi_{+}^{(2)}$ and the linear forcing $\eta_{E, L F,+}^{(2)}$ [cf. Eq. (5)]. For the latter, we obtain from substituting Eqs. (21) and (22) into Eq. (11):

$$
\begin{aligned}
\eta_{E, \mathrm{LF},+}^{(2)}= & \frac{k_{0} A_{1}\left(X_{1}, Y_{1}\right)^{2}}{2} \cos \left(2 \varphi_{1}\right)+\frac{k_{0} A_{2}\left(X_{2}, Y_{2}\right)^{2}}{2} \\
& \times \cos \left(2 \varphi_{2}\right)-\frac{k_{0} A_{1}\left(X_{1}, Y_{1}\right) A_{2}\left(X_{2}, Y_{2}\right)}{2} \\
& \times \cos (\Delta \theta) \cos \left(\varphi_{1}+\varphi_{2}\right) .
\end{aligned}
$$

Substituting Eqs. (21), (22), and (25) into Eq. (4) gives to leading order for $\phi_{+}^{(2)}$

$$
\begin{aligned}
\phi_{E,+}^{(2)}= & -\frac{2[1-\cos (\Delta \theta)] \omega_{0} A_{1}\left(X_{1}, Y_{1}\right) A_{2}\left(X_{2}, Y_{2}\right)}{4-\sqrt{2+2 \cos (\Delta \theta)} \tanh \left(k_{z} d\right)} \\
& \times \frac{\cosh \left[k_{z}(z+d)\right]}{\cosh \left(k_{z} d\right)} \sin \left(\varphi_{1}+\varphi_{2}\right),
\end{aligned}
$$

where $k_{z} \equiv[2+2 \cos (\Delta \theta)]^{1 / 2} k_{0}$. It is clear from Eq. (26) that the potential only has superharmonics in the case of crossing $(\Delta \theta \neq 0)$. Substituting Eq. (26) into Eq. (5) combined with Eq. (25) gives the total Eulerian superharmonic second-order free surface elevation. In nondimensional form, we thus obtain at the crossing point (i.e., $\tilde{\eta}^{(2)} \equiv \eta^{(2)}(x=0, y=0) /\left(k_{0} a_{0}^{2} / 2\right)$, with $A_{1}\left(X_{1}=0\right.$, $\left.\left.Y_{1}=0\right)=A_{2}\left(X_{2}=0, Y_{2}=0\right)=a_{0} / 2\right)$,

$$
\begin{aligned}
\tilde{\eta}_{E,+}^{(2)}= & \frac{1}{4} \cos \left(2 \varphi_{1}\right)+\frac{1}{4} \cos \left(2 \varphi_{2}\right)+\left\{\frac{1}{4}[3-\cos (\Delta \theta)]\right. \\
& \left.-\frac{2[1-\cos (\Delta \theta)]}{4-\left(k_{z} / k_{0}\right) \tanh \left(k_{z} d\right)}\right\} \cos \left(\varphi_{1}+\varphi_{2}\right),
\end{aligned}
$$

where $k_{z} \equiv[2+2 \cos (\Delta \theta)]^{1 / 2} k_{0}$ as before. When two waves travel in the same direction $(\Delta \theta=0)$ and have the same phase $\left(\mu_{0} \equiv \mu_{1}=\mu_{2}\right)$, we obtain $\tilde{\eta}_{E,+}^{(2)}=\cos \left(2 \varphi_{0}\right)$, as for the case of a weakly spread group [cf. Eq. (15)]. When two waves travel in the opposite direction $(\Delta \theta=$ $\left.180^{\circ}\right)$ and have the same phase $\left(\mu_{0} \equiv \mu_{1}=\mu_{2}\right)$, we obtain $\tilde{\eta}_{E,+}^{(2)}=(1 / 2) \cos \left(2 \varphi_{0}\right)$, which is equivalent to the superharmonic terms derived for standing waves by Tadjbakhsh and Keller [1960, their Eq. (46)] (see also Rayleigh 1915; Penney and Price 1952).

\section{2) LAGRANGIAN}

Using the linear solutions for two crossing groups Eqs. (21) and (22) to evaluate the Lagrangian second-order 
correction defined in Eq. (9) gives in nondimensional form [i.e., $\tilde{\eta}^{(2)} \equiv \eta^{(2)}(x=0, y=0) /\left(k_{0} a_{0}^{2} / 2\right)$, with $A_{1}\left(X_{1}=\right.$ $\left.\left.\left.0, Y_{1}=0\right)=A_{2}\left(X_{2}=0, Y_{2}=0\right)=a_{0} / 2\right)\right]$,

$$
\begin{aligned}
\Delta \tilde{\eta}_{L}^{(2)}= & \frac{1}{2}-\frac{1}{4} \cos \left(2 \varphi_{1}\right)-\frac{1}{4} \cos \left(2 \varphi_{2}\right) \\
& +\frac{1}{2} \cos (\Delta \theta)\left[\cos \left(\varphi_{1}-\varphi_{2}\right)-\cos \left(\varphi_{1}+\varphi_{2}\right)\right] .
\end{aligned}
$$

When combined with the Eulerian free surface Eq. (27), the total Lagrangian second-order superharmonic contribution is given by

$$
\begin{aligned}
\eta_{L,+}^{(2)}= & {[1-\cos (\Delta \theta)] } \\
& \times\left[\frac{3}{4}-\frac{2}{4-\sqrt{2+2 \cos (\Delta \theta)}}\right] \cos \left(\varphi_{1}+\varphi_{2}\right) .
\end{aligned}
$$

which is only nonzero when waves $\operatorname{cross}(\Delta \theta \neq 0)$, because the superharmonics due to self interaction are not present in the Lagrangian signal.

If we consider only mean terms, we obtain the total Lagrangian second-order subharmonic contribution,

$$
\eta_{L,-}^{(2)}=\tilde{\eta}_{E, \mathrm{SD}}^{(2)}+\frac{1}{2}+\frac{1}{4}[1+\cos (\Delta \theta)] \cos \left(\mu_{1}-\mu_{2}\right),
$$

which decays with increasing crossing angle.

\section{3) STOKES DRIFT VELOCITY AND LAGRANGIAN FREQUENCY}

To estimate the change in the Lagrangian frequency, we obtain the Stokes drift from Eq. (18) and evaluate the component traveling in the $x$ direction at the crossing point $(x=0, y=0)$ and at $z=0$,

$$
u_{S}=\frac{1}{4} a_{0}^{2} k_{0} \omega_{0}\left\{[1+\cos (\Delta \theta)]+2 \cos ^{4}(\Delta \theta / 2)\right\},
$$

where we have set $A_{1}\left(X_{1}=0, Y_{1}=0\right)=A_{2}\left(X_{2}=0, Y_{2}=\right.$ $0)=a_{0} / 2$. For $\Delta \theta=0$, we recover the Stokes drift of a weakly spread group [Eq. (19)] and for $\Delta \theta=180^{\circ}$, the Stokes drift is zero. The corresponding Doppler-shifted Lagrangian frequency is

$$
\omega_{L, 0}=\omega_{0}\left(1-\frac{1}{4} a_{0}^{2} k_{0}^{2}\left\{[1+\cos (\Delta \theta)]+2 \cos ^{4}(\Delta \theta / 2)\right\}\right) .
$$

\section{e. A single strongly spread wave group}

When the degree of spreading becomes large, the model for a weakly spread group, in which directional spreading is captured by the modulation (in the $y$ direction) of a planar carrier wave (traveling in the $x$ direction), becomes invalid. To examine what is predicted for an SSG, we consider the continuous generalization of Eqs. (6) and (7), in which the directional and frequency (wavenumber magnitude) distributions are assumed to be independent, a standard assumption. In polar coordinates, $r=\left(x^{2}+y^{2}\right)^{1 / 2}$ and $\theta_{p}=\operatorname{atan}(y / x)$, we obtain for the linear free surface

$$
\begin{aligned}
\eta^{(1)}= & \int_{-\infty}^{\infty} \int_{-\pi}^{\pi} \Omega(\theta) \hat{\eta}(k) \cos \left[k r \cos \left(\theta-\theta_{p}\right)\right. \\
& -\omega t+\mu] d \theta d k,
\end{aligned}
$$

and the corresponding linear potential

$$
\begin{aligned}
\phi^{(1)}= & \int_{-\infty}^{\infty} \int_{-\pi}^{\pi} \frac{\omega}{k} \Omega(\theta) \hat{\eta}(k) e^{k z} \sin \left[k r \cos \left(\theta-\theta_{p}\right)\right. \\
& -\omega t+\mu] d \theta d k,
\end{aligned}
$$

where $k=|\mathbf{k}|$ and $\omega(k)$ and $\mu(k)$. We will now consider the limit of a spectrum that is narrow-banded in frequency (wavenumber magnitude) but has arbitrary directional spreading, so that Eqs. (33) and (34) become

$$
\eta^{(1)}=a_{0} \int_{-\pi}^{\pi} \Omega(\theta) \cos \left[k_{0} r \cos \left(\theta-\theta_{p}\right)-\omega_{0} t+\mu_{0}\right] d \theta
$$

and

$\phi^{(1)}=\frac{a_{0} \omega_{0}}{k_{0}} \int_{-\pi}^{\pi} \Omega(\theta) e^{k_{0} z} \sin \left[k_{0} r \cos \left(\theta-\theta_{p}\right)-\omega_{0} t+\mu_{0}\right] d \theta$,

where $\omega_{0}$ and $k_{0}$ are the frequency and wavenumber of the carrier wave. We have not retained any of the group structure, so that Eqs. (35) and (36) present crossing periodic wave trains. To enable closed-form solutions where possible, we will make use of a wrapped normal spreading distribution centered around $\theta=\theta_{0}$ with characteristic spreading width $\sigma_{\theta}$ :

$$
\Omega(\theta)=\frac{1}{\sqrt{2 \pi} \sigma_{\theta}} \sum_{n=-\infty}^{\infty} \exp \left[-\frac{\left(\theta-\theta_{0}+2 \pi n\right)^{2}}{2 \sigma_{\theta}^{2}}\right] .
$$

\section{1) EULERIAN}

At the subharmonic level, the second-order surface is again made up from an Eulerian setdown $\eta_{E, \mathrm{SD}}^{(2)} \equiv-(1 / g) \partial \phi_{E,-}^{(2)} / \partial t$ and a cross-wave term $\eta_{E, \mathrm{CW}}^{(2)} \equiv$ $\eta_{E, \mathrm{LF},-}^{(2)}$ [Eq. (11)] [cf. Eq. (5)]. As for the crossing wave groups, the Eulerian setdown term is driven by the return flow, and its magnitude will depend inversely on 
depth $k_{0} d$ and thus be comparatively small for the deep-water conditions $\left(k_{0} d \gg 1\right)$ considered here. Because of this and since the setdown term can only be evaluated in the form of a triple integral that is only marginally less cumbersome than the multichromatic second-order theory reviewed in section $2 b$, we do not evaluate it here.
The linear forcing term $\eta_{E \text { LF }}^{(2)}$ is not directly affected by depth, and we can find an expression for a narrow-banded frequency distribution by substituting Eqs. (35) and (36) into Eq. (11). Retaining both sub- and superharmonic terms, we obtain at the point $r=0$ and after nondimensionalization [i.e., $\tilde{\eta}^{(2)} \equiv$ $\left.\eta^{(2)}(r=0) /\left(k_{0} a_{0}^{2} / 2\right)\right]$

$$
\tilde{\eta}_{E, \mathrm{LF}}^{(2)}=\frac{1+3 \cos \left(2 \varphi_{0}\right)}{2} \int_{-\pi}^{\pi} \int_{-\pi}^{\pi} \Omega\left(\theta_{1}\right) \Omega\left(\theta_{2}\right) d \theta_{1} d \theta_{2}-\frac{1+\cos \left(2 \varphi_{0}\right)}{2} \int_{-\pi}^{\pi} \int_{-\pi}^{\pi} \Omega\left(\theta_{1}\right) \Omega\left(\theta_{2}\right) \cos \left(\theta_{1}-\theta_{2}\right) d \theta_{1} d \theta_{2},
$$

where $\varphi_{0}=\varphi_{0}(r=0)=-\omega_{0} t+\mu_{0}$. For the wrapped normal distribution Eq. (37), the integrals in Eq. (38) can be evaluated explicitly to give

$$
\tilde{\eta}_{E, \mathrm{LF}}^{(2)}=\frac{1}{2}\left(1-e^{-\sigma_{\theta}^{2}}\right)+\frac{1}{2}\left(3-e^{-\sigma_{\theta}^{2}}\right) \cos \left(2 \varphi_{0}\right),
$$

where we can confirm, in the limit $\sigma_{\theta} \rightarrow 0$, that the subharmonic term vanishes and the superharmonic term approaches its weakly spread counterpart Eq. (12).

Considering only subharmonic terms, for a strongly spread wave group we thus have

$$
\tilde{\eta}_{E,-}^{(2)}=\tilde{\eta}_{E, \mathrm{SD}}^{(2)}+\frac{1}{2}\left(1-e^{-\sigma_{\theta}^{2}}\right) .
$$

From comparison with the weakly spread group [Eq. (15), for which $\left.\tilde{\eta}_{E, \mathrm{SD}}^{(2)}=-1 /\left[2 k_{0} d(1+R)\right]\right\}$, it is evident that nonweak directional spreading introduces a setup of the Eulerian subharmonics free surface, as is predicted for crossing groups [see McAllister et al. (2018) and section $2 \mathrm{~d}]$. This setup vanishes in the limit $\sigma_{\theta} \rightarrow 0$, increases monotonically with the degree of spreading $\sigma_{\theta}$, and reaches a maximum for axisymmetric waves $\sigma_{\theta} \rightarrow \infty$.

Considering only superharmonic terms, it is possible to solve Laplace subject to Eq. (4) and the bottom boundary condition to give

$$
\begin{aligned}
\phi_{E,+}^{(2)}= & -\omega_{0} a_{0}^{2} \int_{-\pi}^{\pi} \int_{-\pi}^{\pi} \frac{\cosh \left[k_{z}(z+d)\right]}{\cosh \left(k_{z} d\right)} \frac{2[1-\cos (\Delta \theta)]}{4-\left(k_{z} / k_{0}\right) \tanh \left(k_{z} d\right)} \\
& \times \sin \left[k_{0} r \cos \left(\theta_{1}-\theta_{p}\right)+k_{0} r \cos \left(\theta_{1}-\theta_{p}\right)\right. \\
& \left.-2 \omega_{0} t+2 \mu_{0}\right] \Omega\left(\theta_{1}\right) \Omega\left(\theta_{2}\right) d \theta_{1} d \theta_{2}
\end{aligned}
$$

where $\Delta \theta \equiv \theta_{2}-\theta_{1}$ and $k_{z} \equiv[2+2 \cos (\Delta \theta)]^{1 / 2} k_{0}$. We note that superharmonics only appear for nonzero spreading. We can then obtain for the superharmonic free surface elevation at second order in nondimensional form

$$
\tilde{\eta}_{E,+}^{(2)}=\left[\frac{3}{2}+F\left(k_{0} d, \sigma_{\theta}\right)-\frac{1}{2} e^{-\sigma_{\theta}^{2}}\right] \cos \left(2 \varphi_{0}\right),
$$

where, for the wrapped normal distribution Eq. (37), the function $F\left(k_{0} d, \sigma_{\theta}\right)$ is given by

$$
F=\frac{-2}{\sqrt{\pi} \sigma_{\theta}} \int_{-\infty}^{\infty} e^{-\left(\Delta \theta / \sigma_{\theta}\right)^{2} / 4} \frac{1-\cos (\Delta \theta)}{4-\left(k_{z} / k_{0}\right) \tanh \left(k_{z} d\right)} d \Delta \theta,
$$

with $k_{z} \equiv[2+2 \cos (\Delta \theta)]^{1 / 2} k_{0}$ as before. We can recover the limit $\tilde{\eta}_{E,+}^{(2)}=\cos \left(2 \varphi_{0}\right)$ for $\sigma_{\theta} \rightarrow 0(F \rightarrow 0)$ [cf. Eq. (15)]. In the limit of an axisymmetric wave $\left(\sigma_{\theta} \rightarrow \infty\right)$, we obtain a small in-phase contribution to the free surface by the superharmonic of $\tilde{\eta}_{E,+}^{(2)} \approx 0.19 \cos \left(2 \varphi_{0}\right)$ in the limit $k_{0} d \rightarrow \infty(F \approx-1.31$, from numerical integration $)$.

\section{2) LAGRANGIAN}

Using the linear solutions for a strongly spread group Eqs. (35) and (36) to evaluate the Lagrangian secondorder correction defined in Eq. (9) gives in nondimensional form [i.e., $\tilde{\eta}^{(2)} \equiv \eta^{(2)}(r=0) /\left(k_{0} a_{0}^{2} / 2\right)$ ]

$$
\begin{aligned}
\Delta \tilde{\eta}_{L}= & \int_{-\pi}^{\pi} \int_{-\pi}^{\pi} \Omega\left(\theta_{2}\right) \Omega\left(\theta_{1}\right) \cos \left(\theta_{1}-\theta_{2}\right) \\
& \times\left[1-\cos \left(2 \varphi_{0}\right)\right] d \theta_{2} d \theta_{2},
\end{aligned}
$$

which can be evaluated explicitly for the wrapped normal distribution Eq. (37):

$$
\Delta \tilde{\eta}_{L}^{(2)}=e^{-\sigma_{\theta}^{2}}\left[1-\cos \left(2 \varphi_{0}\right)\right] .
$$

The total Lagrangian free surface elevation for a strongly spread group is

$$
\begin{aligned}
\tilde{\eta}_{L}^{(2)}= & \tilde{\eta}_{E, \mathrm{SD}}^{(2)}+\frac{1}{2}\left(1+e^{-\sigma_{\theta}^{2}}\right)\left[-\frac{1}{2}+F\left(k_{0} d, \sigma_{\theta}\right)\right. \\
& \left.+\frac{1}{2} e^{-\sigma_{\theta}^{2}}\right] \cos \left(2 \varphi_{0}\right)
\end{aligned}
$$

\section{3) STOKES DRIFT VELOCITY AND LAGRANGIAN FREQUENCY}

To estimate the change in the Lagrangian frequency, we obtain the Stokes drift from Eq. (18) and evaluate 
the component traveling in the $x$ direction at the crossing point $\left(r=0, \theta_{p}=0\right)$ and at $z=0$ for the wrapped normal distribution Eq. (37):

$$
u_{S}(r=0, z=0)=\frac{a_{0}^{2} k_{0} \omega_{0}}{4}\left(3 e^{-\sigma_{\theta}^{2} / 2}+e^{-5 \sigma_{\theta}^{2} / 2}\right) .
$$

We thus obtain for the Lagrangian wave frequency

$$
\omega_{L, 0}=\omega_{0}\left[1-\frac{a_{0}^{2} k_{0}^{2}}{4}\left(3 e^{-\sigma_{\theta}^{2} / 2}+e^{-5 \sigma_{\theta}^{2} / 2}\right)\right],
$$

which becomes equal to the analogous result for the weakly spread group Eq. (20) in the limit $\sigma_{\theta} \rightarrow 0$. As $\sigma_{\theta} \rightarrow \infty$, the Stokes drift disappears, and the Lagrangian period becomes equal to the Eulerian pe$\operatorname{riod}\left(\omega_{L, 0} \rightarrow \omega_{0}\right)$.

\section{f. Comparison with multicomponent second-order theory}

Table 1 summarizes the results for the three cases considered above. We will now compare our approximate second-order expressions with exact multicomponent second-order theory from HJ16 for narrow-banded spectra [section $2 \mathrm{f}(1)$ ] and for realistic broadbanded spectra [section $2 \mathrm{f}(2)]$.

\section{1) NARROW-BANDED SPECTRA}

Figures $1 \mathrm{a}-\mathrm{c}$ compare the normalized amplitude $\left(\tilde{a}^{(2)}=a^{(2)} /\left(k_{0} a_{0}^{2} / 2\right)\right.$ with $a^{(2)}$ denoting the amplitude of $\left.\eta^{(2)}\right)$ of sub- and superharmonics predicted using exact second-order theory (solid lines) to our approximate expressions (dotted lines) for a single wave group as a function of the degree of directional spreading $\sigma_{\theta}$. Red lines show the magnitude of the Eulerian components $\tilde{a}_{E}^{(2)}$, gray lines show the Lagrangian correction $\tilde{a}_{\Delta L}^{(2)}$, and blue lines show the magnitude of the Lagrangian components $\tilde{a}_{L}^{(2)}$. We choose the water depth $k_{0} d=3.94$ to correspond to our experiments, and the discrepancy between our approximate solutions (dotted lines) and exact second-order theory (solid lines) in Fig. 1 comes from excluding the setdown in our approximate solutions. Figures $1 \mathrm{~d}-\mathrm{f}$ are analogous to Figs. $1 \mathrm{a}-\mathrm{c}$ but illustrate the second-order amplitude for two crossing wave groups as a function of the crossing angle $\Delta \theta$. As expected, our approximate expressions compare well to exact theory for wave groups with a narrow underlying spectrum $\left(\Delta k / k_{0}=0.05\right)$ given explicitly by

$$
\hat{\eta}(k)=\frac{a_{0}}{\sqrt{2 \pi} \Delta k} \exp \left[-\frac{1}{2}\left(\frac{k-k_{0}}{\Delta k}\right)\right] .
$$

Examining trends in Fig. 1, in the limit of no spreading or crossing $\left(\sigma_{\theta} \rightarrow 0, \Delta \theta \rightarrow 0\right)$, the amplitude of the
Eulerian second-order subharmonics (Figs. 1a,e) is small and equal to the setdown $\tilde{\eta}_{E \text { SD }}^{(2)}$, which vanishes for truly deep water $\left(k_{0} d \rightarrow \infty\right)$. The Lagrangian correction is at its maximum. As the degree of spreading or the crossing angle is increased, a positive Eulerian setup forms and the Lagrangian correction tends to zero. Therefore, in the limit $\left(\sigma_{\theta} \rightarrow \infty ; \Delta \theta \rightarrow 180\right)$, Eulerian and Lagrangian second-order components become equal. The same is true for superharmonic components (Figs. 1c,f), which also tend toward each other in the limit of large spreading or head-on crossing $(\Delta \theta \rightarrow 180)$. However, without spreading or crossing, Eulerian superharmonic components are at their largest and are completely canceled out by the Lagrangian correction, which is negative. When combined, the sub- and superharmonic components of the Lagrangian correction completely cancel out (Figs. 1c,f). Thus, for narrow-banded wave groups on deep water, the total second-order contribution to crest height for Eulerian and Lagrangian measurements is equal.

\section{2) REALISTIC BROAD-BANDED SPECTRA}

Our approximate expressions for second-order contribution to crest height are derived using the assumption that the spectra that underlie our wave groups are narrow banded in frequency. In reality, ocean waves typically follow relatively broadbanded frequency distributions with a slowly decaying high-frequency tail. To estimate how well our approximate expressions perform for realistic broadbanded spectra, we compare these with predictions made using exact second-order theory for focused wave groups based on a JONSWAP spectrum (Hasselmann et al. 1980):

$$
\hat{\eta}(\omega)=\beta \omega^{n} \exp \left[\frac{n}{4}\left(\frac{\omega_{p}}{\omega}\right)^{4}\right] \gamma^{\exp \left[-\left(\omega-\omega_{p}\right)^{2} /\left(2 \sigma^{2} \omega_{p}^{2}\right)\right]},
$$

where $\gamma$ is the peak enhancement factor, $\omega_{p}$ is the peak frequency, $n$ is the slope of the high-frequency tail, $\sigma\left(\omega<\omega_{p}\right)=0.07$ and $\sigma\left(\omega>\omega_{p}\right)=0.09$, and $\beta$ is a scale parameter that is adjusted to give the desired amplitude. We emphasize again that we set the amplitude spectrum to be proportional to the energy spectrum in Eq. (50), because it provides a good (and commonly used) approximation to the shape of extreme waves in the ocean (Lindgren 1970; Boccotti 1983; Phillips et al. 1993).

In Fig. 2, we compare predictions made using our approximate expressions Eqs. (29) and (30) (dotted lines) to predictions made using exact second-order theory for wave groups based on Gaussian $\left(\Delta k=0.6 \mathrm{~m}^{-1}\right.$; dashed lines) and JONSWAP spectra $(\gamma=3.3$ and $n=-5$; solid lines). The relatively broadbanded Gaussian spectrum $\left(\Delta k / k_{0}=0.30\right)$ is what we use in our 


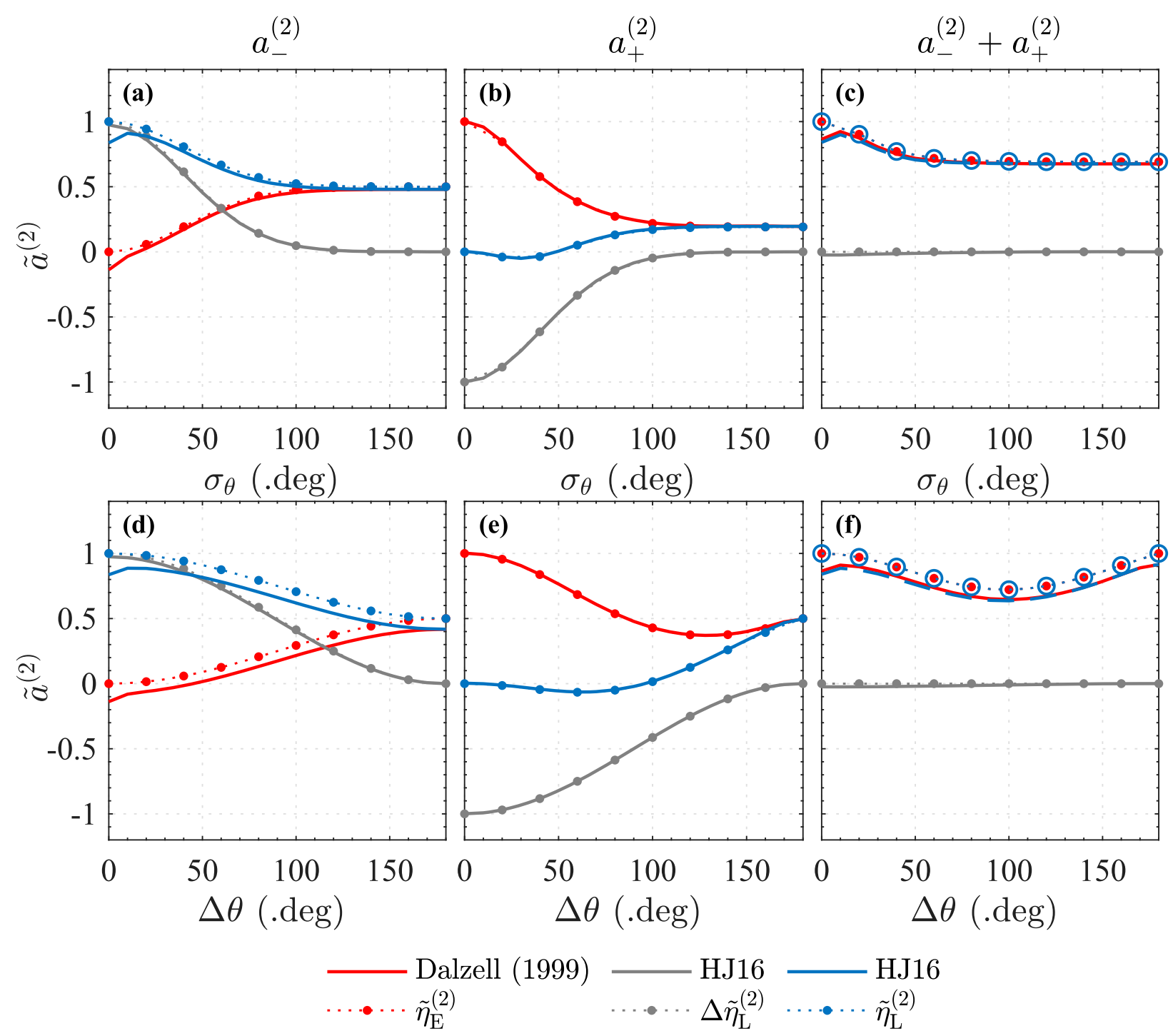

FIG. 1. Comparison of our approximate expressions (dotted lines with filled circles) to exact multichromatic (solid lines) second-order (a),(d) subharmonic and (b),(e) superharmonic bound wave components and (c),(f) their sum, for (top) directionally spread and (bottom) crossing focused wave groups based on narrow Gaussian spectra (spectral width $\Delta k=0.1 \mathrm{~m}^{-1}$ ) on a water depth $d=2 \mathrm{~m}$, with peak wavenumber $k_{0}=1.97 \mathrm{~m}^{-1}$ and for focused crests $\left(\mu_{0}=\mu_{1}=\mu_{2}=0\right)$. The red, gray, and blue lines respectively denote the magnitude $\tilde{a}^{(2)}=a^{(2)} /\left(k_{0} a_{0}^{2} / 2\right)$ of the Eulerian components $\eta_{E}^{(2)}$, the Lagrangian corrections $\Delta \eta_{L}^{(2)}$, and the Lagrangian components $\eta_{L}^{(2)}$. Open circles are used in (c) and (f) for visual clarity.

experiments. For the relatively broadbanded Gaussian spectrum, our approximate expressions for the superharmonic components follow predictions made using exact second-order well. However, when considering subharmonic components, a clear offset is observed. This offset occurs because we do not include in our approximate expressions the Eulerian setdown term $\tilde{\eta}_{E, S D}$ [without crossing and spreading, we can readily estimate $\tilde{\eta}_{E, \mathrm{SD}}=-0.13$ for $k_{0} d=3.9$ from Eq. (15)]. For the JONSWAP spectrum in Fig. 2, our approximate expressions perform very well with only a small error from not including the setdown. When the same calculations are performed for an increased water depth $\left(k_{0} d=7.9\right)$, this offset is reduced (dot-dashed lines). Our approximate expressions become more accurate for realistic spectra when the water depth becomes truly deep and the setdown can be entirely ignored, as expected. Examining directionally spread groups gives qualitatively and quantitatively similar results.

\section{Experimental method}

In this section, we first introduce our experimental setup (section 3a). We then detail the input parameters 

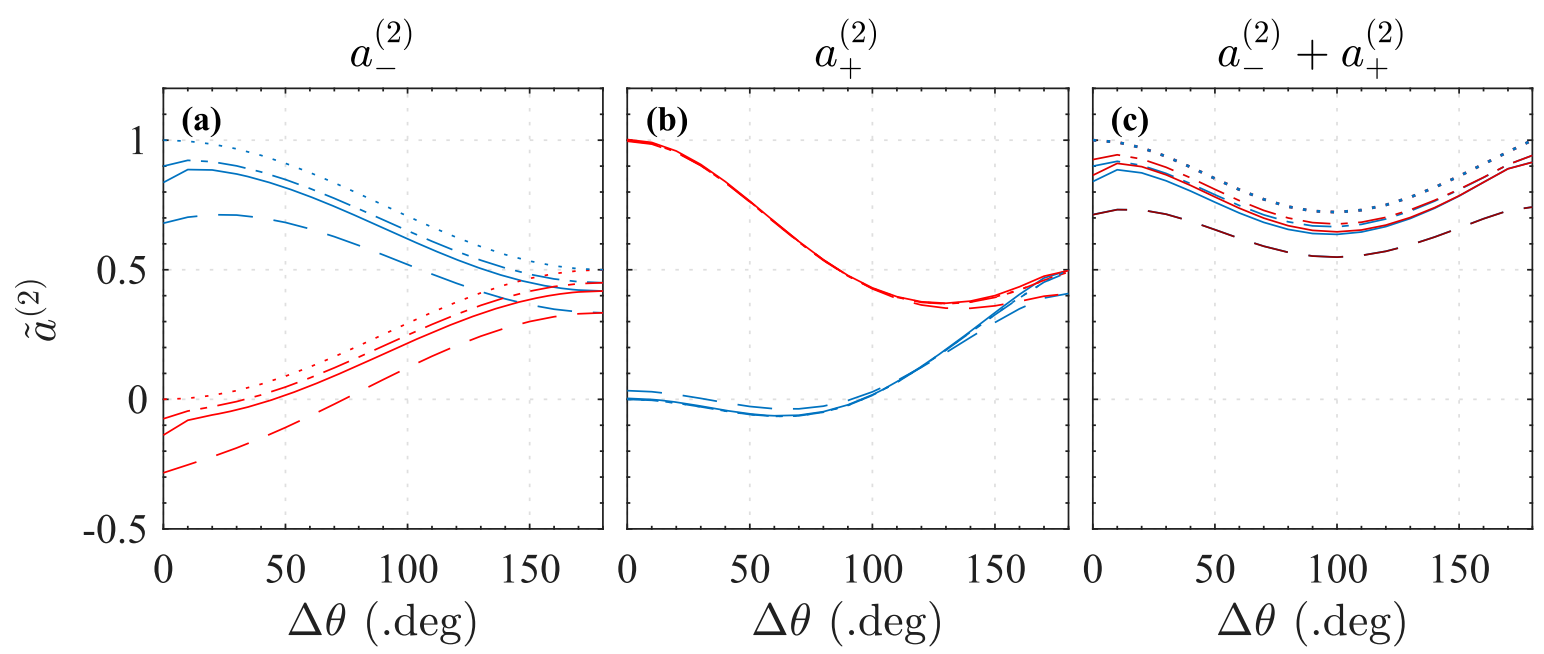

$$
\text { - - } \eta_{\mathrm{L}}, \Delta k=0.6 \mathrm{~m}^{-1}-\eta_{\mathrm{E}}, \Delta k=0.6 \mathrm{~m}^{-1}-\eta_{\mathrm{E}}-\eta_{\mathrm{E}}, \Delta
$$
$\eta_{\mathrm{L}}$ JONSWAP $----\eta_{\mathrm{L}}$ $\eta_{\mathrm{L}}$ JONSWAP $d=4 \mathrm{~m}$ $\cdots \cdots \cdot \eta_{\mathrm{L}}, \Delta k \rightarrow 0$

FIG. 2. Amplitude of Eulerian (red) and Lagrangian (blue) second-order bound wave components from exact multichromatic secondorder theory for crossing focused wave groups with Gaussian $\left(\Delta k=0.6 \mathrm{~m}^{-1}\right.$, representative of our experiments $)$ and JONSWAP spectra $(\gamma=3.3 ; n=-5)$, as shown by solid and dashed lines, respectively. The groups have input degrees of directional spreading of $\sigma_{\theta}=20^{\circ}$, peak frequencies of $0.7 \mathrm{~Hz}$, and a depth of $2.0 \mathrm{~m}\left(k_{0} d=3.9\right)$. The dot-dashed lines show predictions using exact multichromatic secondorder theory for a JONSWAP spectrum with an increased depth of $d=4.0 \mathrm{~m}$. Dotted lines show predictions using our narrow-banded approximations. Shown are the amplitudes of (a) subharmonic, (b) superharmonic, and (c) total bound wave components as a function of the crossing angle $\Delta \theta$.

we use for our experiments (section $3 b$ ) and the process used to estimate the spectral and directional parameters achieved in the tank (section 3c). In section 3d we discuss sources of measurement error and the repeatability of our experiments.

\section{a. Experimental setup}

The experiments presented herein were carried out in the FloWave Ocean Energy Research Facility at the University of Edinburgh. This is a circular wave basin, which is $2 \mathrm{~m}$ deep and surrounded by 168 actively absorbing force-feedback wavemakers, which allows for the creation of waves that travel in any direction. With the exception of the Lagrangian wave measurements, the experimental setup and procedure follows exactly that of McAllister et al. (2018). Eulerian measurements of free surface elevation were made using resistive wave gauges, which were calibrated at the start of each day of testing. Any Eulerian measurements presented herein are at the intended point of focus in the center of the $\operatorname{tank}(x=0, y=0)$.

Although waves are in principle absorbed by the wavemakers, this process is not perfect, and reflections may still occur. To avoid reflections, we set the run time of our experiments to $32 \mathrm{~s}$ and allowed for $10 \mathrm{~min}$ of settling time between each experiment to ensure the dissipation of any background motion in the tank.
To measure free surface elevation, wave-following buoys first measure acceleration, which is then integrated twice to give displacement. These displacements are projected onto the coordinate system of heave, north, and east displacements. In our experiments, we assume that there is no error in this process [see van Essen et al. (2018) and Raghukumar et al. (2019) for a discussion of this assumption], and consider the position of our model buoy to be what a wave buoy in the ocean would measure.

We measure the position of our model buoy using a Qualisys motion-tracking system, consisting of four calibrated infrared cameras located above the tank, which simultaneously track the position of the buoy. To aid its detection, the spherical model buoy was covered in a coating that reflects the specific band of infrared light emitted and recorded by the cameras. The buoy's position in each set of images is then triangulated to measure its global position within the tank.

\section{1) Model Lagrangian BuOY}

Wave-following measurement buoys are in general spherical to avoid sensitivity to yaw, pitch, and roll motion. These properties are measured by other types of, typically larger, weather buoys, which often measure a range of "metocean" conditions, such as wind speed in addition to wave height. Because the latter of these two 
types of buoy behavior is far from Lagrangian, they will not be considered in this study.

Wave-following buoys typically have diameters in the range of $0.5-1 \mathrm{~m}$ and operate on water depths from tens to thousands of meters, in waves of $1-30 \mathrm{~m}$ in height and $1-20 \mathrm{~s}$ in period. This wide range of operating conditions presents a challenge for scaling. If a floating body's characteristic length (diameter) is of the same order as the length of the waves incident on it, its motion will cause diffraction and radiation. To correctly scale these effects, similar ratios of buoy diameter to incident wavelength, $D / \lambda$, must be maintained between our model and a buoy operating in the ocean. The density of our buoy was chosen to be approximately one-half that of the water, to match that of a real measurement buoy.

The diameter of a buoy will also affect the forces that dominate its motion. If the fluid velocity relative to a buoy's size is large, drag forces will dominate and, conversely, if velocity is low, inertial forces will dominate. The Keulegan-Carpenter number, which can be approximated for a stationary body in periodic linear waves as $K_{C}=2 \pi a_{0} / D$, is often used to characterize the relative importance of these two forces. A wavefollowing buoy will not be stationary, and if purely Lagrangian its velocity relative to the fluid will tend to zero. This makes it difficult to correctly evaluate the $K_{C}$ number a priori. In light of this, we do not directly evaluate the $K_{C}$. However, it is worthwhile checking that the ratio of our buoy's diameter to wave amplitude $D / a_{0}$ is of a similar order to that of a real buoy. Also, the water depth $k_{0} d$ on which a buoy operates will affect the kinematics of the incident waves.

Our study is motivated by extreme ocean waves. Our experiments have $k_{0} d \approx 4$, representative of deep-water conditions (typically, $k_{0} d \gtrsim 3$ ). Increasing water depth further will not affect the resulting linear kinematics and will, at second order, only affect the magnitude of the setdown, which is already small (cf. Figs. 1 and 2), and very slightly the Eulerian superharmonic for large crossing angles and degrees of spreading. Mooring designs will change with increasing water depth, but we do not consider the effects of mooring herein. Extreme ocean waves will typically have long periods in the range of $10-20 \mathrm{~s}$; for a $1.0-\mathrm{m}$ buoy on deep water, this gives the range $D / \lambda=1.6 \times 10^{-3}-6.4 \times 10^{-2}$. The diameter of our model buoy is $0.07 \mathrm{~m}$, and the peak period of the waves we test is $1.43 \mathrm{~s}$, which gives $D / \lambda=3.6 \times 10^{-2}$. Therefore, at both scales, $D / \lambda \ll 1$, meaning diffraction, radiation, and hydrodynamic resonance will not play a significant role. Extreme wave crest heights or amplitudes may range between 5 and $15 \mathrm{~m}$; for a $1.0-\mathrm{m}$ buoy this gives ratios of $a_{0} / D=5-15$. The wave amplitudes that we test result in ratios of around $a_{0} / D=2.14-2.86$.
These values are somewhat lower than the most extreme waves. Testing with a smaller buoy was not possible, because reliably tracking the motion of a very small buoy becomes difficult and the auxiliary mooring forces discussed below become dominant and start to affect buoy motion.

Ideally, to perform a comparison of predicted Lagrangian motion to the actual Lagrangian motion of our buoy, the buoy would have no mooring. However, an auxiliary mooring was necessary to achieve readily repeatable experiments. Additional experiments were carried out with no auxiliary mooring, and it was found that the mooring had no observable effect on the measured free surface elevation including bound wave components. We emphasize that the effect of a mooring on buoy motion in a realistic configuration is not the objective of this study.

\section{b. Matrix of experiments and input parameters}

To recreate conditions that are representative of extreme events that occur in the oceans, we examine focused wave groups (Lindgren 1970; Boccotti 1983; Phillips et al. 1993). We carry out two categories of experiments (see Table 2): experiments with (weakly and strongly) spread wave groups [section $3 b(1)]$ and experiments with crossing wave groups [section $3 b(2)$ ]. In the first category, we vary the degree of directional spreading for a single group; in the second category, we vary the angle at which two wave groups cross.

For all experiments, we produced focused wave groups based on an input Gaussian amplitude distribution in wavenumber $k=|\mathbf{k}|$,

$$
\hat{\eta}(k)=\frac{a_{0}}{\sqrt{2 \pi} \Delta k} \exp \left[-\frac{1}{2}\left(\frac{k-k_{0}}{\Delta k}\right)\right]
$$

with peak wavenumber $k_{0}=1.97 \mathrm{~m}^{-1}$ (based on a frequency of $0.7 \mathrm{~Hz}$ ) and standard deviation $\Delta k=0.6 \mathrm{~m}^{-1}$. The value $a_{0}$ is adjusted to give the desired wave steepness. We carry out experiments at two steepness, $a_{0} k_{0}=0.3$ and 0.4 ; these high steepnesses were chosen to replicate extreme ocean waves and generate large and hence readily measurable second-order bound components without causing wave breaking. We define our inputs using this form to allow for consistency with the second-order theory presented in section 2 . The input parameters we use are a chosen to fit within the optimum generation range of the wavemakers, while achieving a relatively spatially compact wave group to minimize the influence of reflections and error waves associated with linear wave paddle motion. We define directional spreading using a Gaussian amplitude distribution in angle $\theta$, 
TABLE 2. Experimental matrix (a dagger symbol represents experiments carried out without auxiliary mooring).

\begin{tabular}{ccccr}
\hline \hline Category & $D(\mathrm{~m})$ & $a_{0}(\mathrm{~m})$ & $\sigma_{\theta}\left({ }^{\circ}\right)$ & $\Delta \theta\left(^{\circ}\right)$ \\
\hline Spreading & 0.07 & 0.15 & $0,10,20^{\dagger}, 30,40^{\dagger}, 50$, and $\infty^{\dagger}$ & 0 \\
Crossing & 0.07 & 0.20 & 20,40, and $\infty$ & 0 \\
\end{tabular}

$$
\Omega(\theta)=\frac{\Omega_{0}}{\sqrt{2 \pi} \sigma_{\theta}} \sum_{n=-N}^{N} \exp \left[-\frac{\left(\theta-\theta_{0}+2 \pi n\right)^{2}}{2 \sigma_{\theta}^{2}}\right],
$$

where we set the normalizing coefficient $\Omega_{0}$ so that the sum of $\Omega(\theta)$ over the finite number of components $N$ is unity. For crossing wave groups, two amplitude distributions with different values of $\theta_{0}$ are superimposed. Note that our value of $\sigma_{\theta}$ applies to an amplitude distribution and not to the commonly used energy spectrum for which the degree of spreading will be equal to $\sigma_{\theta} / \sqrt{2}$.

\section{1) EXPERIMENTS WITH SPREAD WAVE GROUPS}

We first examine single directionally spread wave groups (see Table 2$)$. We vary spreading from unidirectional $\left(\sigma_{\theta}=0^{\circ}\right)$ to axisymmetric $\left(\sigma_{\theta} \rightarrow \infty\right)$ at an input steepness of $a_{0} k_{0}=0.3$. We repeat experiments for an unmoored buoy at spreading widths of $20^{\circ}, 40^{\circ}$, and $\infty^{\circ}$. We also carry out additional experiments for our moored buoy at increased steepness $a_{0} k_{0}=0.4$.

\section{2) EXPERIMENTS WITH CROSSING WAVE GROUPS}

In addition to single wave groups, we examine the Lagrangian measurement of extreme crossing wave groups (see Table 2). For these experiments, we set the directional spreading of each group $\sigma_{\theta}=20^{\circ}$ and vary the angle at which the two wave groups cross $\Delta \theta$ between $0^{\circ}$ and $180^{\circ}$ at $45^{\circ}$ intervals. The steepness of the two combined wave groups was set as $a_{0} k_{0}=0.3$.

\section{c. Estimation of spectral and directional parameters}

To verify the test conditions achieved in the tank, we estimate the spectral parameters of our measured free surface elevation. This is carried out for both Eulerian and Lagrangian measurements (see Table 3 ). To estimate the linear amplitude $a_{0}^{\star}$ of each wave group, we take the zeroth moment of the measured linear spectrum, because it provides an estimate of amplitude that is less sensitive to focusing than using crest amplitude. For both the Eulerian and the Lagrangian measurements, we estimate peak frequency $\omega_{0}^{\star}$ and quantify the spectral bandwidth $\Delta \omega^{\star}$ as the variance of the measured spectra.

To estimate the degree of directional spreading achieved for each experiment $\sigma_{\theta}^{\star}$, we use Eulerian measurements made with an array of 14 wave gauges. Because of the nonergodic nature of our experiments, we cannot apply conventional methods of spreading estimation. Instead, we use a least squares approach combined with linear wave theory to determine the degree of spreading $\sigma_{\theta}^{\star}$ (see McAllister et al. 2018). Input and estimated parameters are reported in Table 3.

TABLE 3. Input and estimated spectral parameters, where the estimated spectral parameters are denoted by a star, a dagger symbol represents tests that were repeated 3 times, and an em dash represents tests that were omitted.

\begin{tabular}{|c|c|c|c|c|c|c|c|c|c|c|c|c|}
\hline \multirow[b]{2}{*}{ Test no. } & \multicolumn{5}{|c|}{ Input } & \multicolumn{4}{|c|}{ Eulerian } & \multicolumn{3}{|c|}{ Lagrangian } \\
\hline & $\sigma_{\theta}\left({ }^{\circ}\right)$ & $\Delta \theta\left(^{\circ}\right)$ & $\omega_{0}\left(\operatorname{rad~s}^{-1}\right)$ & $a_{0}(\mathrm{~m})$ & $\Delta k\left(\mathrm{~m}^{-1}\right)$ & $\sigma_{\theta}^{\star}\left({ }^{\circ}\right)$ & $\begin{array}{c}\omega_{E, 0}^{\star} \\
\left(\operatorname{rad~s}^{-1}\right)\end{array}$ & $a_{E, 0}^{\star}(\mathrm{m})$ & $\begin{array}{c}\Delta \omega_{E}^{\star} \\
\left(\operatorname{rad~s}^{-1}\right)\end{array}$ & $\begin{array}{c}\omega_{L, 0}^{\star} \\
\left(\operatorname{rad~s}^{-1}\right)\end{array}$ & $a_{L, 0}^{\star}(\mathrm{m})$ & $\begin{array}{c}\Delta \omega_{L}^{\star} \\
\left(\operatorname{rad~s}^{-1}\right)\end{array}$ \\
\hline 1.1 & 0 & 0 & 4.4 & 0.3 & 0.6 & 0 & 4.19 & 0.157 & 1.18 & 4.05 & 0.159 & 1.17 \\
\hline 1.2 & 10 & 0 & 4.4 & 0.3 & 0.6 & 7.8 & 4.18 & 0.137 & 1.11 & 4.08 & 0.137 & 1.10 \\
\hline $1.3^{\dagger}$ & 20 & 0 & 4.4 & 0.3 & 0.6 & 15.7 & 4.18 & 0.123 & 1.05 & 4.10 & 0.121 & 0.99 \\
\hline 1.4 & 30 & 0 & 4.4 & 0.3 & 0.6 & 28.0 & 4.25 & 0.127 & 1.07 & 4.17 & 0.134 & 1.10 \\
\hline $1.5^{\dagger}$ & 40 & 0 & 4.4 & 0.3 & 0.6 & 38.0 & 4.25 & 0.127 & 1.08 & 4.19 & 0.134 & 1.10 \\
\hline 1.6 & 50 & 0 & 4.4 & 0.3 & 0.6 & 47.6 & 4.25 & 0.126 & 1.06 & 4.20 & 0.13 & 1.09 \\
\hline $1.7^{\dagger}$ & $\infty$ & 0 & 4.4 & 0.3 & 0.6 & - & - & - & - & 4.24 & 0.121 & 0.62 \\
\hline 2.1 & 0 & 0 & 4.4 & 0.4 & 0.6 & 0 & 3.98 & 0.206 & 1.37 & 3.89 & 0.212 & 1.48 \\
\hline 2.2 & 20 & 0 & 4.4 & 0.4 & 0.6 & 17.6 & 4.13 & 0.165 & 1.17 & 4.00 & 0.165 & 1.21 \\
\hline 2.3 & 40 & 0 & 4.4 & 0.4 & 0.6 & 38.6 & 4.25 & 0.168 & 1.08 & 4.14 & 0.176 & 1.08 \\
\hline 2.4 & $\infty$ & 0 & 4.4 & 0.4 & 0.6 & $\infty$ & 4.24 & 0.169 & 1.04 & 4.24 & 0.170 & 1.08 \\
\hline 3.1 & 20 & 45 & 4.4 & 0.3 & 0.6 & 17.8 & 4.25 & 0.129 & 1.09 & 4.17 & 0.133 & 1.02 \\
\hline 3.2 & 20 & 90 & 4.4 & 0.3 & 0.6 & 18.6 & 4.25 & 0.129 & 1.09 & 4.21 & 0.133 & 1.02 \\
\hline 3.3 & 20 & 135 & 4.4 & 0.3 & 0.6 & 19.1 & 4.23 & 0.131 & 1.09 & 4.23 & 0.134 & 1.00 \\
\hline 3.4 & 20 & 180 & 4.4 & 0.3 & 0.6 & 18.6 & 4.24 & 0.130 & 1.04 & 4.24 & 0.136 & 1.01 \\
\hline
\end{tabular}




\section{d. Measurement error and repeatability}

To quantify the sources of error affecting our comparison between experiments and theory, we examine the role of the various sources of error and uncertainty involved in our experiments. For the Eulerian measurements, McAllister et al. (2018) found that the leading source of error was most likely associated with wave gauge calibration $\left( \pm \Delta \eta_{E} \approx\right.$ $0.4 \mathrm{~mm}$ ), with residual tank motion and experiment repeatability resulting in errors of approximately \pm 0.025 and $\pm 0.023 \mathrm{~mm}$, respectively ( 2 standard deviations). For the Eulerian measurements, McAllister et al. (2018) noted that tests carried out at larger amplitudes reduce the variation between repeat experiments. We carry out the majority of our experiments at the larger of the two amplitudes tested in McAllister et al. (2018).

Our Lagrangian measurements will be subject to similar sources of error. To understand these sources of error, we assess the repeatability of these measurements (error measure I) and compute the accuracy of the Qualisys motion-tracking system (error measures II and III). The repeatability of our experiments was extremely high, with 2 times the standard deviation of repeat experiments ranging from \pm 0.08 to $\pm 1.00 \mathrm{~mm}$. The error calculated when calibrating the Qualisys system was slightly larger at approximately $\pm 0.5 \mathrm{~mm}$, when measuring a distance of $300 \mathrm{~mm}$, which is approximately the height of our waves. The leading source of error was associated with locating the buoy's position during experiments, where timevarying residual values ranged from around \pm 0.5 to $\pm 1.5 \mathrm{~mm}$. At every time stamp when the Qualisys system measures the position of the centroid of our model buoy, a residual value is returned. This value represents the diameter of a sphere, inside which all the centroids identified by each camera pair lie. We use these residual values measured during each experiment to calculate error bounds for the results we present herein.

\section{Results}

In this section, we present the results of our experiments and compare Eulerian and Lagrangian measurements of directionally spread and crossing wave groups. In section $4 \mathrm{a}$, we examine differences in the measured linear free surface elevation. In section $4 b$, we then examine the second-order sub- and superharmonics and their contribution to measured crest height; we also compare our results with predictions based on the theory we present in section 2. Throughout, because of the near-perfect agreement, we will refer to measurements obtained from the model buoy as Lagrangian measurements.

\section{a. Linear free surface elevation}

We begin by comparing the linear free surface elevation measured by our Lagrangian buoy and Eulerian wave gauges [see McAllister et al. (2018) for details of our linearization procedure]. Figures $3 \mathrm{a}$ and $3 \mathrm{~b}$ show the linear free surface elevation measured by our Lagrangian buoy $\eta_{L}^{(1)}$ (red dashed lines) with the corresponding Eulerian measurements $\eta_{E}^{(1)}$ (black solid lines) for single directionally spread wave groups as the degree of spreading is increased from narrowly spread to near-axisymmetric. This figure shows the steepest wave groups we created, for which the input steepness was $a_{0} k_{0}=0.4$. Figures $3 \mathrm{c}$ and $3 \mathrm{~d}$ illustrate changes to the linear spectra $\hat{\eta}^{(1)}$; these changes are also quantified in Table 3. In Fig. 3, for narrow directional spreading $\sigma_{\theta}$, the period of the Lagrangian measurements is increased; this is visible when considering the measured free surface elevation and the resulting amplitude spectrum.

Performing a direct comparison between values of Lagrangian frequency predicted by our approximate expressions and those measured during our experiments is challenging. The relativity broadbanded wave groups we create have an envelope that varies quickly, and hence so does the Lagrangian frequency, making it difficult to measure reliably. Instead, we include vertical dashed and dotted lines in Figs. 3c and $3 \mathrm{~d}$ that show the measured peak frequency and corresponding approximate Lagrangian peak frequency obtained from Eq. (48). The comparison is intended to be qualitative. In narrowly spread conditions (Fig. 3c), our expression predicts a down-shift of the Lagrangian frequency, which compares well to the down-shift we observe in our experiments. On the contrary, in the limit of $\sigma_{\theta} \rightarrow \infty$ (Fig. 3d) no Doppler shift is observed, which is also in line with our predictions. In general, as spreading is increased, either for a single group or two crossing waves, the difference between the Lagrangian and Eulerian period tends to zero.

Figure 4a compares the amplitude of the waves measured by our model buoy $a_{L}$ with their Eulerian equivalent $a_{E}$ measured at the position of intended focus $(x=0, y=0)$ across the full range of conditions we test (see Table 2). The red symbols show the full crest amplitude measured by each device, and the black symbols show the linearized crest amplitude. In general, for the smaller waves, both are approximately equal. However, for the larger-amplitude waves, some 

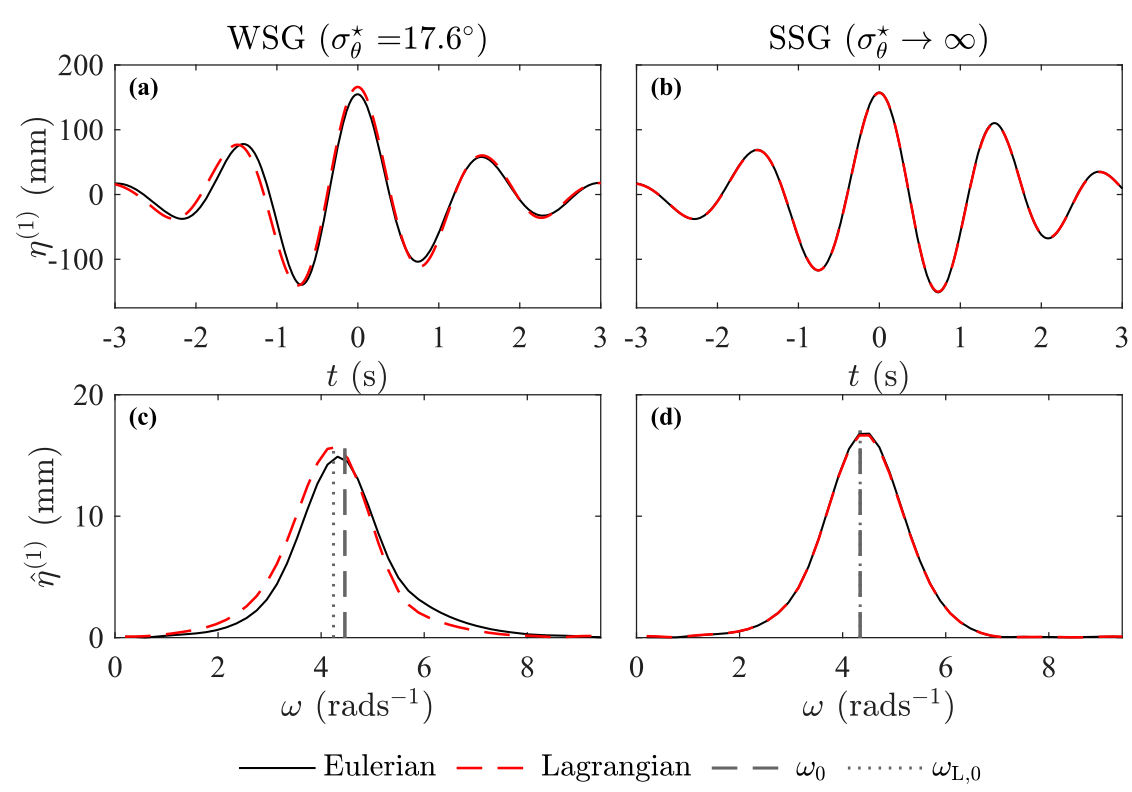

FIG. 3. Eulerian (black lines) and Lagrangian (red dashed lines) linear free surface elevation $\eta^{(1)}$ for (a) a WSG $\left(\sigma_{\theta}=20^{\circ}\right)$ and (b) an SSG $\left(\sigma_{\theta} \rightarrow \infty\right)$ and (c),(d) their respective amplitude spectra $\hat{\eta}^{(1)}$ for steepness $a_{0} k_{0}=0.4$. The Lagrangian peak frequency $\omega_{L, 0}$ is calculated using Eq. (48), noting that we obtain $\omega_{L, 0} \rightarrow \omega_{0}$ for $\sigma_{\theta} \rightarrow \infty$.

of the Lagrangian measurements are greater than their Eulerian counterparts.

In Fig. 4b, we compare the ratio of the linearized Lagrangian and Eulerian amplitudes $a_{L}^{(1)} / a_{E}^{(1)}$ as a function of the degree of directional spreading $\sigma_{\theta}$ (circles and triangles) and crossing angle $\Delta \theta$ (crosses). Note that the values of $a^{(1)}$ that we present are measured crest amplitudes, unlike values of $a_{0}^{\star}$, which are based on the zeroth spectral moment. A small increase in measured Lagrangian amplitude (relative to Eulerian) is only observed for unidirectional and very narrowly spread wave groups. We measured waves of equivalent amplitudes with large degrees of spreading and found no difference between Eulerian and Lagrangian measurements in that case (shown by the triangles in the upper portion of Fig. 4a that lie on the line $a_{L}=a_{E}$ ). Therefore, we hypothesize this difference in linear amplitude is a phenomenon associated with the horizontal transport of the buoy beyond what is predicted by any second-order theory. The wave groups that we create are broadbanded in frequency, meaning that they disperse and change shape significantly and rapidly as they propagate across the wave tank. When our wave groups are unidirectional or narrowly spread, the resulting horizontal transport of the buoy is at its largest. It is also the case that the most steep and narrowly spread wave groups we create focus slightly downstream of our central wave gauge. Hence, as Stokes drift causes the buoy to be transported downstream, it measures a slightly larger wave group than the Eulerian gauge, which is fixed at $x=0, y=0$.

\section{b. Second-order free surface elevation}

Figures 5 and 6 present the second-order sub- (top row) and superharmonic (bottom row) bound waves from our experiments. In each panel, the black solid line shows the measured Eulerian free surface elevation at the intended point of focus $(x=0, y=0)$, and the black dashed line shows the corresponding Lagrangian free surface elevation for the same incident wave group. The red dashed and blue dotted lines show predictions made using exact Eulerian and Lagrangian multicomponent second-order theory, respectively. Both sets of predictions are based on the measured Eulerian linear free surface elevation $\eta_{E}^{(1)}$.

\section{1) Directionally SPREAD WAVE GROUPS}

In Fig. 5, we consider directionally spread wave groups and we vary the degree of directional spreading. From left to right in the figure, the input value of $\sigma_{\theta}$ is increased from $0^{\circ}$ to $50^{\circ}$ in $10^{\circ}$ increments; the actual degree of spreading measured in the tank $\sigma_{\theta}^{\star}$ is displayed above each panel. The input amplitude $a_{0}$ of these wave groups was set to $0.15 \mathrm{~m}$, giving a steepness of $a_{0} k_{0}=0.3$.

For both sub- and superharmonic bound waves, the Eulerian measurements agree remarkably well for all degrees of spreading shown. As $\sigma_{\theta}$ initially increases, the amplitudes of both sub- and superharmonic bound 


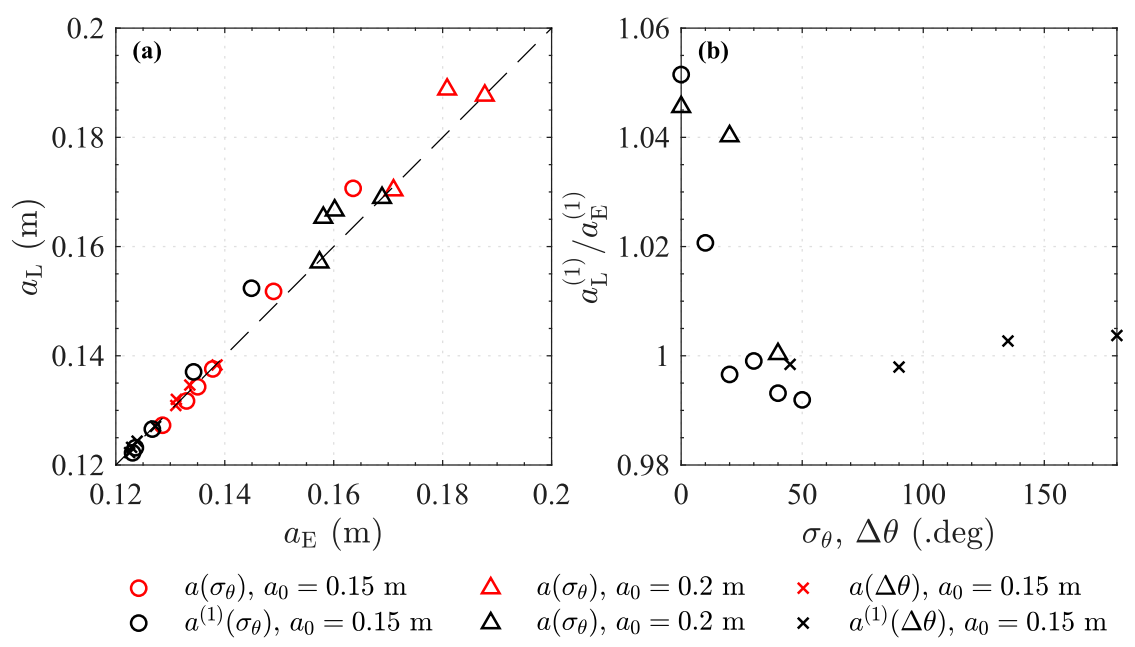

FIG. 4. Measured wave crest amplitude for directionally spread wave groups with input steepness $a_{0} k_{0}=0.3$ (circles) and $a_{0} k_{0}=0.4$ (triangles) and crossing wave groups $\left(a_{0} k_{0}=0.3\right.$; crosses). (a) A comparison of the crest amplitude $a_{L}$ measured by our Lagrangian buoy with Eulerian measurements $a_{E}$, with red symbols showing fully nonlinear measured crest amplitudes and black symbols showing linearized crest heights. (b) Variation of the ratio of linearized amplitudes $a_{L}^{(1)} / a_{E}^{(1)}$ as a function of directional spreading and crossing angle.

wave components decrease. However, as spreading is further increased, the Eulerian subharmonic components become large and positive, corresponding to the setup studied in McAllister et al. (2018) (see also Fig. 7b). Excluding the unidirectional case in Fig. 5a, the measured Lagrangian bound wave components also match theoretical predictions very well. This discrepancy is a result of the increased linear amplitude measured by the buoy discussed above, as calculations are based on the linear Eulerian measurement. For all degrees of spreading, we observe a positive setup when considering the Lagrangian subharmonic bound wave components, which decreases in amplitude as the degree of spreading is increased. The amplitude of the measured Lagrangian superharmonic bound wave components is negligibly small for these experiments, as predicted for a perfectly
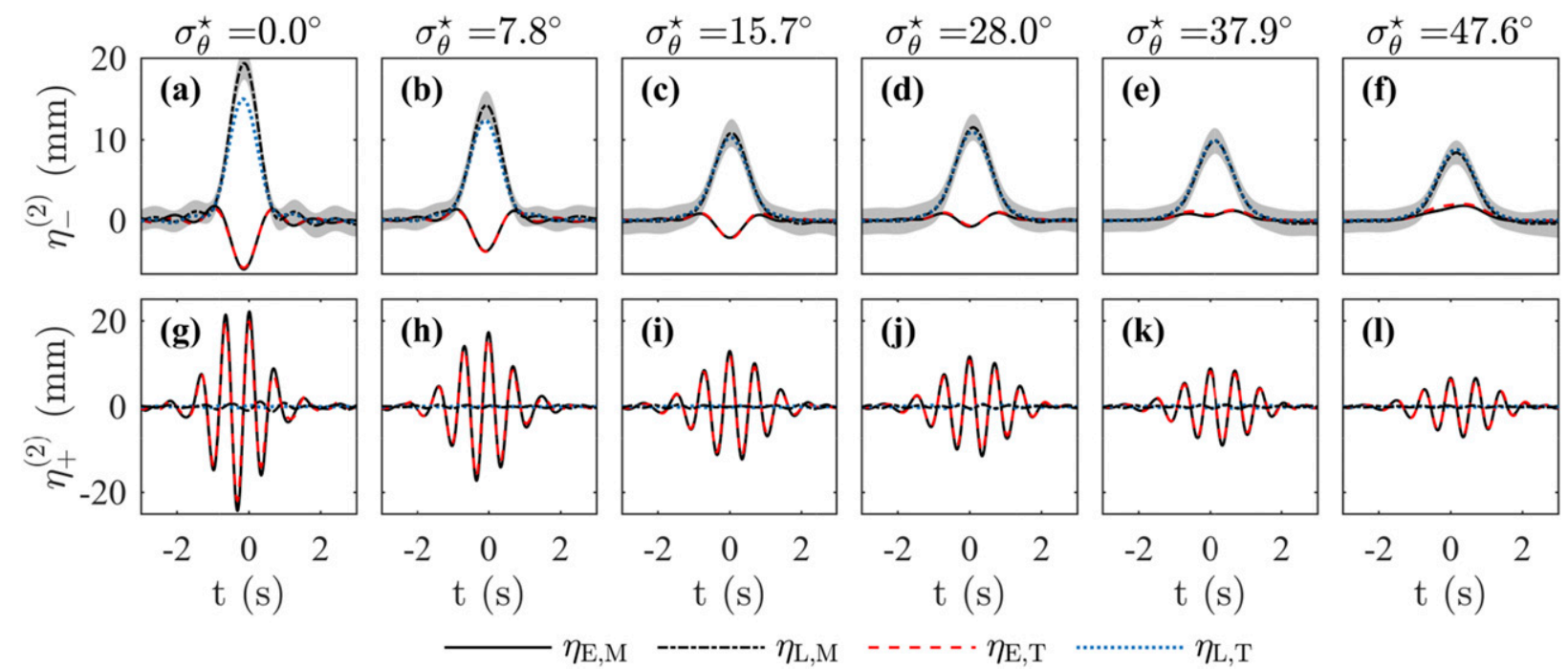

FIG. 5. Second-order (a)-(f) subharmonic and (g)-(l) superharmonic bound wave components for weakly and strongly spread wave groups with input degrees of directional spreading $\sigma_{\theta}=0^{\circ}-50^{\circ}$ in increments of $10^{\circ}$ and input steepness $a_{0} k_{0}=0.3$. Estimated degrees of directional spreading $\sigma_{\theta}^{\star}$ are given at the top. Black solid lines show Eulerian measurements, and black dot-dashed lines show Lagrangian measurements. Red dashed lines and blue dotted lines show predictions made using Eulerian and Lagrangian second-order theory, respectively. Gray envelopes show measurement error bounds. 


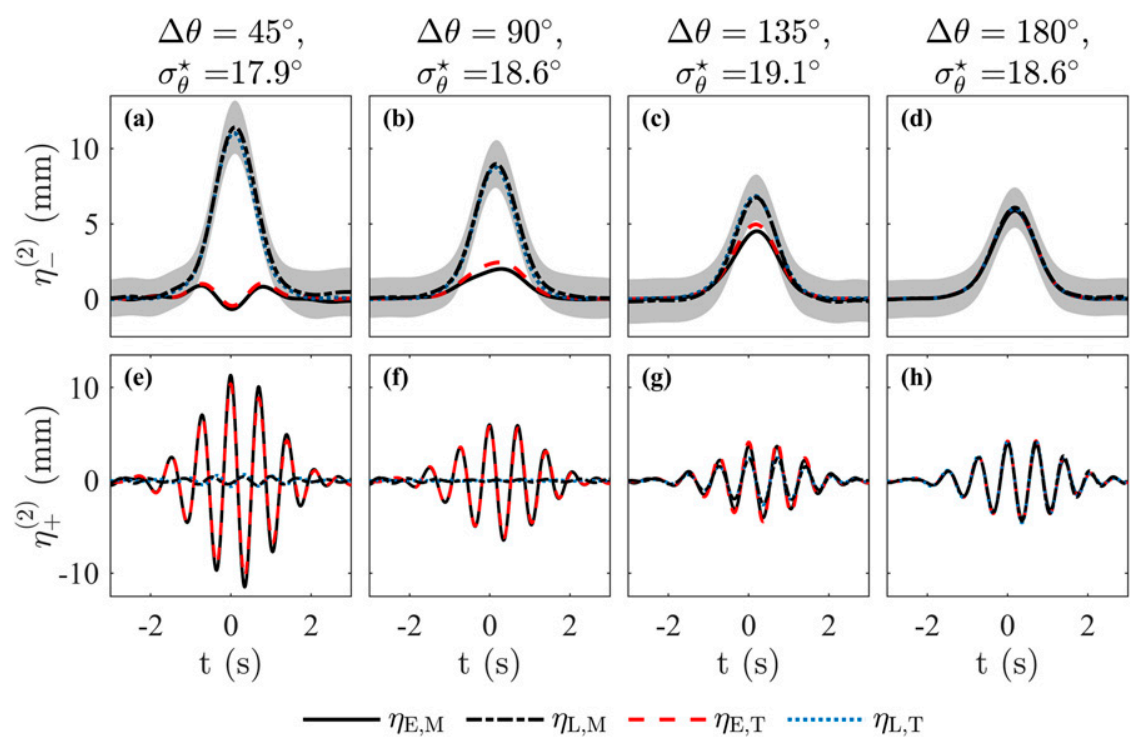

FIG. 6. Second-order (a)-(d) subharmonic and (e)-(h) superharmonic bound wave components for two crossing wave groups with input degrees of directional spreading $\sigma_{\theta}=20^{\circ}$, input steepness $a_{0} k_{0}=0.3$, and crossing angle between the two wave groups $\Delta \theta=45^{\circ}, 90^{\circ}, 135^{\circ}$, and $180^{\circ}$. Estimated degrees of directional spreading $\sigma_{\theta}^{\star}$ are given at the top. Black solid lines show Eulerian measurements, and black dot-dashed lines show Lagrangian measurements. Red dashed lines and blue dotted lines show predictions made using Eulerian and Lagrangian second-order theory, respectively. Gray envelopes show measurement error bounds.

Lagrangian observer for up to moderate degrees of directional spreading.

\section{2) Crossing WAVE Groups}

In Fig. 6, we consider two crossing wave groups, each with input directional spread $\sigma_{\theta}=20^{\circ}$ and a combined input steepness of $a_{0} k_{0}=0.3$. We vary the angle at which the two wave groups cross. From left to right in the figure, the crossing angle is increased from $0^{\circ}$ to $180^{\circ}$ in increments of $45^{\circ}$. For these experiments, theoretical predictions match our experimental observations very well. At low crossing angles, the Lagrangian second-order bound wave components are similar to those observed in Fig. 5. When the waves cross at large angles, the Lagrangian bound wave components tend to their Eulerian equivalents; this also occurs for a single directionally spread wave group when $\sigma_{\theta} \rightarrow \infty$ (cf. Figs. 7b-d).

When waves cross at large angles or are highly directionally spread, the horizontal velocity they induce cancels out at the point of focus. Therefore, there is no horizontal motion, and the Lagrangian surface elevation recorded by a buoy is equivalent to the Eulerian surface elevation. At moderate crossing angles and degrees of spreading, Lagrangian measurements lie between two extremes, where on the one hand we essentially have standing waves with horizontal motion of the buoy tending to zero $\left(\eta_{E}=\right.$ $\left.\eta_{L}\right)$, and on the other hand we have the unidirectional limit where horizontal motion is the largest $\left(\eta_{E} \neq \eta_{L}\right)$.

\section{3) CONTRIBUTION TO CREST AMPLITUDE}

In Figs. 7 and 8, we examine the behavior of the Eulerian and Lagrangian second-order bound components as functions of $\sigma_{\theta}$ and $\Delta \theta$ in more detail. In these figures, we present the magnitude of the second-order bound components normalized by the measured wavenumber $k_{0}^{\star}$ and amplitude $a_{0}^{\star}$ (see Table 3 ). By combining sub- and superharmonics, we also consider the total second-order contribution to crest amplitude. The continuous lines in these figures represent theoretical predictions of the magnitude of second-order bound components for perfectly focused Gaussian wave groups with the same parameters as our experiments. When normalized in this way, it becomes clear that the combined Lagrangian bound wave components do not decrease in magnitude monotonically with increasing $\sigma_{\theta}$ or $\Delta \theta$ (cf. Figs. $7 \mathrm{~d}$ and $8 \mathrm{~d}$ ). Furthermore, when combined, the total second-order bound wave contributions of Eulerian and Lagrangian measurements do not differ significantly. Figures $7 \mathrm{a}$ and $8 \mathrm{a}$ illustrate that, under the conditions we test, the second-order motion of our buoy is the same as that of a perfectly Lagrangian observer.

\section{4) TOTAL FREE SURFACE ELEVATION}

Figure 9 compares the total measured Eulerian $\eta_{E}$ (black lines) and Lagrangian $\eta_{L}$ (red dashed lines) free surface elevation for the steepest input waves we created 


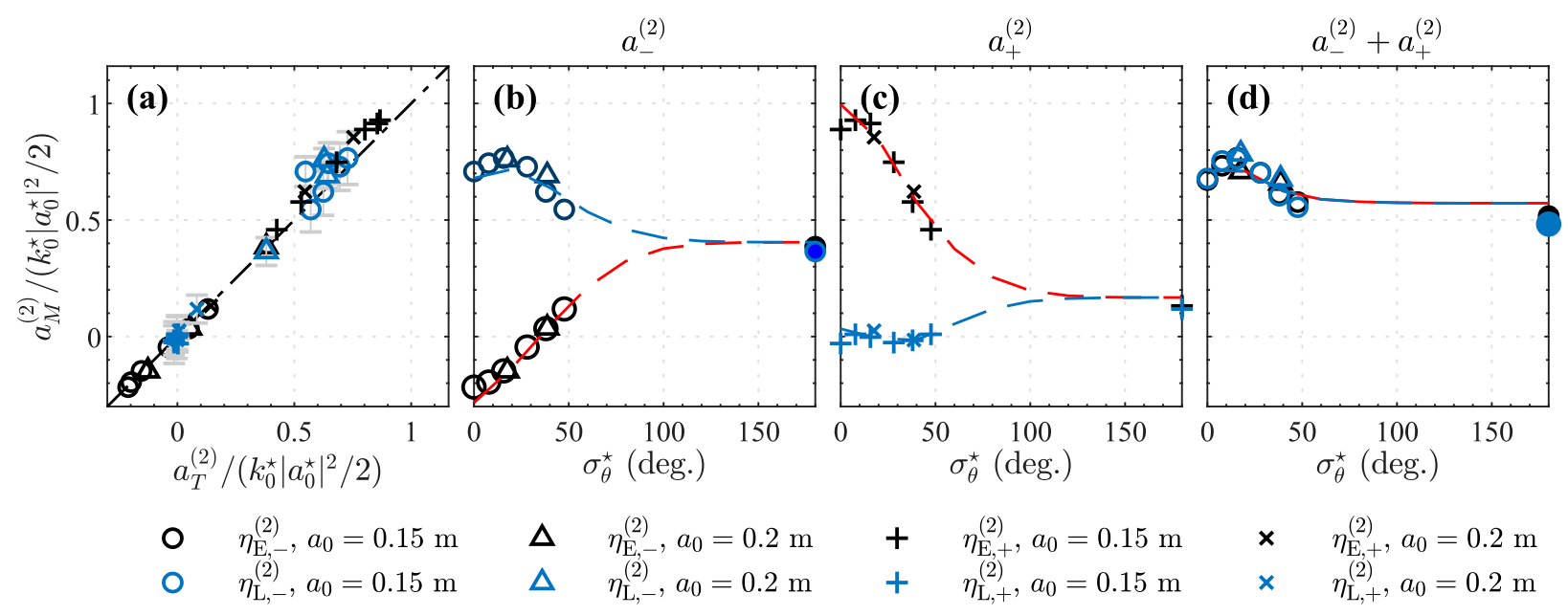

FIG. 7. Amplitudes of Eulerian and Lagrangian second-order bound wave components for weakly and strongly directionally spread focused wave groups. (a) A comparison of theoretical predictions (subscript $T$ ) with the measured (subscript $M$ ) amplitude of both superand subharmonic components (crosses and circles, respectively). Also shown are the amplitudes of (b) subharmonic, (c) superharmonic, and (d) total bound wave components as a function of the estimated degree of directional spreading $\sigma_{\theta}^{\star}$. Throughout, blue symbols represent Lagrangian measurements and black symbols represent Eulerian measurements. Similarly, the blue and red dashed lines represent theoretical Lagrangian and Eulerian predictions, respectively.

$\left(a_{0} k_{0}=0.4\right)$. For steep near-breaking moderately spread wave groups (Figs. 9a,b), we see differences between Eulerian and Lagrangian measurements—namely an apparent broadening of the wave crest. As spreading is further increased (Fig. 9c), we do not observe differences in the measured time series. The observed difference in local wave steepness for moderately spread wave groups occurs as a result of two effects. First, changes to the Lagrangian linear waves makes their period longer than their Eulerian equivalents (cf. Fig. 3). Second, superharmonic components make the measured Eulerian crests narrower and troughs broader. Therefore, although the total second-order contributions to crest amplitude do not differ significantly, for moderate degrees of spreading, the time series of Lagrangian and Eulerian measurements are different.

\section{Conclusions}

Wave measurement buoys are widely used to provide in situ wave measurements. These measurements are used to calibrate ocean models, inform engineering design, and study ocean wave phenomena. Particularly when studying extreme waves, measurements generated by wave buoys are perceived to be less accurate than Eulerian measurements (Dysthe and Müller 2008).

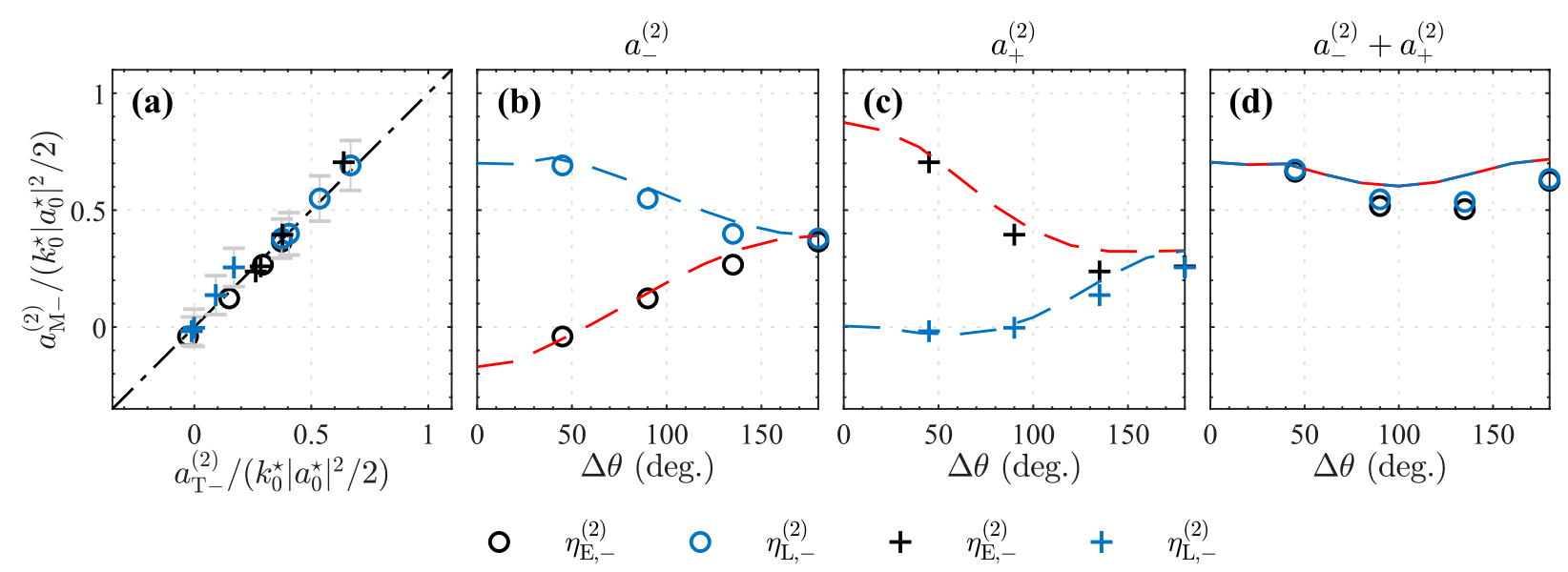

FIG. 8. As in Fig. 7, but for two crossing focused wave groups with input degrees of directional spreading $\sigma_{\theta}=20^{\circ}$ and (b)-(d) as a function of the crossing angle $\Delta \theta$. 


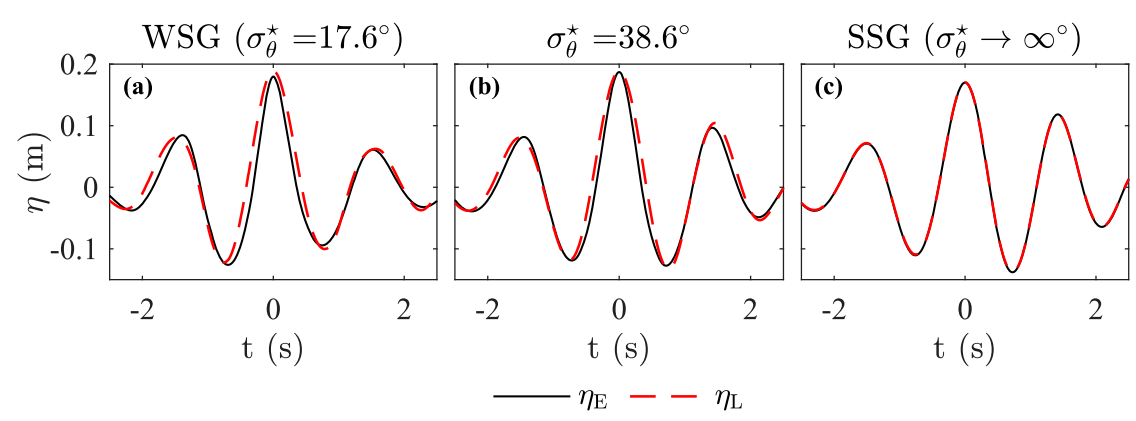

FIG. 9. Comparison of total measured Eulerian (black lines) and Lagrangian (red dashed lines) free surface elevations for (a),(b) WSGs and (c) SSGs of input steepness $a_{0} k_{0}=0.4$.

It should be noted that, in the field, Eulerian wave measurements also fall prey to issues such sea spray and beam scattering that affect their accuracy (Forristall et al. 2004). In the field, as both sensor types are imperfect and are rarely located together, it is very difficult to directly assess their accuracy and identify particular sources error.

In lieu of direct comparison, various explanations are commonly given to explain why buoy measurements may underreport extreme wave crests. It is well understood that as waves become steep, and their behavior nonlinear, Eulerian and Lagrangian free surface measurements become fundamentally different (LonguetHiggins 1986; Srokosz and Longuet-Higgins 1986; HJ16; Grue and Kolaas 2019). Therefore, it is often suggested that differences between Lagrangian and Eulerian measurements may cause the underestimation of extreme waves crest.

In this paper, we have derived a series of approximate second-order expressions that may be used to predict Eulerian and Lagrangian free surface elevation and Stokes drift velocity for extreme waves, which we compare with the exact multicomponent second-order theory of HJ16. The leading-order nonlinear effect of Lagrangian motion is a cancellation of second-order bound superharmonics and a corresponding increase in amplitude of second-order subharmonic components. Although this constitutes a change in the frequency content of the second-order contributions to crest height measured by the two types of device, our theoretical predictions show that in deep water this does not result in a significant change to wave crest amplitude. This phenomenon essentially constitutes a shift of secondorder bound energy from high (superharmonics) to low (subharmonics) frequencies, as identified by HJ16. For devices that measure acceleration, where integration is required to convert to displacement, and data are implicitly high-pass filtered, this shifting of energy content may cause the underestimation of crest height (Marthinsen and Winterstein 1992; Prevosto et al. 2000;
Forristall 2000). If a wave-following buoy behaves in a purely Lagrangian manner, there should be no significant differences in measured wave crests, assuming no error in the measurement acquisition process (see van Essen et al. 2018).

This raises an additional question as to whether wavefollowing buoys do in fact behave in a purely Lagrangian manner. Wave measurement buoys float on the surface, and have densities of approximately half that of seawater and hence may be subject to inertial (Santamaria et al. 2013; Pizzo et al. 2019) and slope sliding effects (Meylan et al. 2015) identified by these authors in different physical contexts. Therefore, wave-following measurement buoys may not behave in a purely Lagrangian manner and, as is commonly perceived, they may avoid extreme wave crests (Krogstad and Barstow 2000).

We have carried out a series of laboratory experiments to assess whether the motion of a scaled wavefollowing buoy in extreme ocean waves is purely Lagrangian and have carried out a direct comparison between Eulerian and buoy measurements of steep directionally spread wave groups at laboratory scale. We have done so in a range of directionally spread conditions, including crossing. The second-order motion of our model buoy compares extremely well to predictions made using existing exact Lagrangian second-order theory (HJ16) and our novel approximate expressions. It is only in the most extreme cases that we test, in which the waves are unidirectional, that we note observable differences. In such cases, we see an apparent modification to the linear waves measured by the buoy, which we believe is a result of the fact that the buoy's displacement becomes large enough for our wave group to have dispersed and changed shape during its measurement. Otherwise, we find that buoy motion at the scale we test is well predicted by approximate and exact Lagrangian second-order theory. In the range of conditions that we test, excluding near-unidirectional conditions as discussed 
above, any deviation from Lagrangian motion of measurement buoys in the field is most likely a result of mooring forces or an artifact of internal signal processing. Future work will address the application of the findings in this paper to random sea states and real buoy data.

Acknowledgments. This project was funded by the EPSRC and Wave Energy Scotland (Grant 24840078 and 24841554). Author van den Bremer was supported by a Royal Academy of Engineering Research Fellowship. The authors thank Mr. E. Nixon, Dr. R. Gabl, and Dr. T. Davey at the FloWave Ocean Energy Research Facility for their assistance in planning and carrying out the experiments.

\section{REFERENCES}

Allender, J., and Coauthors, 1989: The WADIC project: A comprehensive field evaluation of directional wave instrumentation. Ocean Eng., 16, 505-536, https://doi.org/10.1016/ 0029-8018(89)90050-4.

Ashton, I. G. C., and L. Johanning, 2015: On errors in low frequency wave measurements from wave buoys. Ocean Eng., 95, 11-22, https://doi.org/10.1016/j.oceaneng.2014.11.033.

Benetazzo, A., and Coauthors, 2017: On the shape and likelihood of oceanic rogue waves. Sci. Rep., 7, 8276, https://doi.org/ 10.1038/s41598-017-07704-9.

Bidlot, J., D. J. Holmes, P. A. Wittmann, R. Lalbeharry, and H. S. Chen, 2002: Intercomparison of the performance of operational ocean wave forecasting systems with buoy data. Wea. Forecasting, 17, 287-310, https://doi.org/10.1175/1520-0434(2002) 017<0287:IOTPOO > 2.0.CO;2.

Boccotti, P., 1983: Some new results on statistical properties of wind waves. Appl. Ocean Res., 5, 134-140, https://doi.org/ 10.1016/0141-1187(83)90067-6.

Christou, M., P. Tromans, L. Vanderschuren, and K. Ewans, 2009: Second-order crest statistics of realistic sea states. Proc. 11th Int. Workshop on Wave Hindcasting and Forecasting, Halifax, NS, Canada, WMO/UNESCO/IOC/JCOMM, 18-23, http://www.waveworkshop.org/11thWaves/Papers/ Christou_et_al_second_order_crest_statistics.pdf.

Collins, C. O., III, B. Lund, T. Waseda, and H. C. Graber, 2014: On recording sea surface elevation with accelerometer buoys: lessons from ITOP (2010). Ocean Dyn., 64, 895-904, https:// doi.org/10.1007/s10236-014-0732-7.

Dalzell, J. F., 1999: A note on finite depth second-order wave-wave interactions. Appl. Ocean Res., 21, 105-111, https://doi.org/ 10.1016/S0141-1187(99)00008-5.

Dysthe, K. B., and H. E. K. P. Müller, 2008: Oceanic rogue waves. Annu. Rev. Fluid Mech., 40, 287-310, https://doi.org/10.1146/ annurev.fluid.40.111406.102203.

Fedele, F., and F. Arena, 2010: Long-term statistics and extreme waves of sea storms. J. Phys. Oceanogr., 40, 1106-1117, https:// doi.org/10.1175/2009JPO4335.1.

Forristall, G. Z., 2000: Wave crest distributions: Observations and second-order theory. J. Phys. Oceanogr., 30, 1931-1943, https://doi.org/10.1175/1520-0485(2000)030<1931:WCDOAS> 2.0.CO;2.

—, S. F. Barstow, H. E. Krogstad, M. Prevosto, P. H. Taylor, and P. S. Tromans, 2004: Wave crest sensor intercomparison study:
An overview of WACSIS. J. Offshore Mech. Arct., 126, 26-34, https://doi.org/10.1115/1.1641388.

Gemmrich, J., and J. Thomson, 2017: Observations of the shape and group dynamics of rogue waves. Geophys. Res. Lett., $\mathbf{4 4 ,}$ 1823-1830, https://doi.org/10.1002/2016GL072398.

Grue, J., and J. Kolaas, 2019: On the Lagrangian period in steep periodic waves. Water Waves, https://doi.org/10.1007/ s42286-019-00004-x, in press.

Guedes Soares, C. G., Z. Cherneva, and E. M. Antao, 2004: Steepness and asymmetry of the largest waves in storm sea states. Ocean Eng., 31, 1147-1167, https://doi.org/10.1016/j.oceaneng.2003.10.014.

Hasselmann, D. E., M. Dunckel, and J. A. Ewing, 1980: Directional wave spectra observed during JONSWAP 1973. J. Phys. Oceanogr., 10, 1264-1280, https://doi.org/10.1175/1520-0485(1980) 010<1264:DWSODJ > 2.0.CO;2.

Hasselmann, K., 1962: On the non-linear energy transfer in a gravity-wave spectrum Part 1. General theory. J. Fluid Mech., 12, 481-500, https://doi.org/10.1017/S0022112062000373.

Herbers, T. H. C., and T. T. Janssen, 2016: Lagrangian surface wave motion and Stokes drift fluctuations. J. Phys. Oceanogr., 46, 1009-1021, https://doi.org/10.1175/JPO-D-15-0129.1.

_, S. Elgar, and R. T. Guza, 1994: Infragravity-frequency (0.005$0.05 \mathrm{~Hz})$ motions on the shelf. Part I: Forced waves. J. Phys. Oceanogr., 24, 917-927, https://doi.org/10.1175/1520-0485(1994) 024<0917:IFHMOT>2.0.CO;2.

—, P. F. Jessen, T. T. Janssen, D. B. Colbert, and J. H. MacMahan, 2012: Observing ocean surface waves with GPStracked buoys. J. Atmos. Oceanic Technol., 29, 944-959, https://doi.org/10.1175/JTECH-D-11-00128.1.

Holthuijsen, L. H., and T. H. C. Herbers, 1986: Statistics of breaking waves observed as whitecaps in the open sea.J. Phys. Oceanogr., 16, 290-297, https://doi.org/10.1175/1520-0485(1986) 016<0290:SOBWOA $>2.0 . \mathrm{CO}$; .

Janssen, P. A. E. M., B. Hansen, and J. Bidlot, 1997: Verification of the ECMWF wave forecasting system against buoy and altimeter data. Wea. Forecasting, 12, 763-784, https://doi.org/ 10.1175/1520-0434(1997)012<0763:VOTEWF $>2.0 . C O ; 2$.

Krogstad, H. E., and S. F. Barstow, 2000: A unified approach to extreme value analysis of ocean waves. Tenth Int. Offshore and Polar Engineering Conf., Seattle, WA, International Society of Offshore and Polar Engineers, 6 pp.

Lenee-Bluhm, P., R. Paasch, and H. T. Özkan-Haller, 2011: Characterizing the wave energy resource of the US Pacific Northwest. Renew. Energy, 36, 2106-2119, https://doi.org/10.1016/ j.renene.2011.01.016.

Lindgren, G., 1970: Some properties of a normal process near a local maximum. Ann. Math. Stat., 41, 1870-1883, https:// doi.org/10.1214/aoms/1177696688.

Liu, Q., T. Lewis, Y. Zhang, and W. Sheng, 2015: Performance assessment of wave measurements of wave buoys. Int. J. Mar. Energy, 12, 63-76, https://doi.org/10.1016/j.ijome.2015.08.003.

Longuet-Higgins, M. S., 1986: Eulerian and Lagrangian aspects of surface waves. J. Fluid Mech., 173, 683-707, https://doi.org/ 10.1017/S0022112086001325.

, and R. W. Stewart, 1962: Radiation stress and mass transport in gravity waves, with applications to 'surf beats'. J. Fluid Mech., 13, 481-504, https://doi.org/10.1017/S0022112062000877. , and - 1964: Radiation stresses in water waves; a physical discussion, with applications. Deep-Sea Res., 2, 529-562.

Marthinsen, T., and S. R. Winterstein, 1992: On the skewness of random surface waves. Second Int. Offshore and Polar Engineering Conf., San Francisco, CA, International Society of Offshore and Polar Engineers, 7 pp. 
McAllister, M. L., T. A. A. Adcock, P. H. Taylor, and T. S. van den Bremer, 2018: The set-down and set-up of directionally spread and crossing surface gravity wave groups. J. Fluid Mech., 835 , 131-169, https://doi.org/10.1017/jfm.2017.774.

Meylan, M. H., L. J. Yiew, L. G. Bennetts, B. J. French, and G. A. Thomas, 2015: Surge motion of an ice floe in waves: Comparison of a theoretical and an experimental model. Ann. Glaciol., 56, 155-159, https://doi.org/10.3189/2015AoG69A646.

Mork, G., S. Barstow, A. Kabuth, and M. T. Pontes, 2010: Assessing the global wave energy potential. 29th Int. Conf. on Ocean, Offshore and Arctic Engineering, Shanghai, China, American Society of Mechanical Engineers, 447-454, https:// doi.org/10.1115/OMAE2010-20473.

O'Reilly, W. C., T. H. C. Herbers, R. J. Seymour, and R. T. Guza, 1996: A comparison of directional buoy and fixed platform measurements of Pacific swell. J. Atmos. Oceanic Technol., 13, 231-238, https://doi.org/10.1175/1520-0426(1996)013<0231: ACODBA $>2.0 . C O ; 2$.

Okihiro, M., R. T. Guza, and R. J. Seymour, 1992: Bound infragravity waves. J. Geophys. Res., 97, 11 453-11 469, https:// doi.org/10.1029/92JC00270.

Pellet, L., P. Christodoulides, S. Donne, C. J. Bean, and F. Dias, 2017: Pressure induced by the interaction of water waves with nearly equal frequencies and nearly opposite directions. Theor. Appl. Mech. Lett., 7, 138-144, https://doi.org/10.1016/ J.TAML.2017.04.002.

Penney, W. G., and A. T. Price, 1952: Part ii. Finite periodic stationary gravity waves in a perfect liquid. Philos. Trans. Roy. Soc. London, 244A, 254-284, https://doi.org/10.1098/rsta.1952.0004.

Phillips, O. M., D. Gu, and M. Donelan, 1993: Expected structure of extreme waves in a Gaussian sea. Part I: Theory and SWADE buoy measurements. J. Phys. Oceanogr., 23, 992-1000, https://doi.org/10.1175/1520-0485(1993)023<0992: ESOEWI $>2.0 . \mathrm{CO} ; 2$.

Pizzo, N., W. K. Melville, and L. Deike, 2019: Lagrangian transport by nonbreaking and breaking deep-water waves at the ocean surface. J. Phys. Oceanogr., 49, 983-992, https://doi.org/ 10.1175/JPO-D-18-0227.1.

Pontes, M. T., 1998: Assessing the European wave energy resource. J. Offshore Mech. Arct., 120, 226-231, https://doi.org/10.1115/ 1.2829544

Prevosto, M., H. E. Krogstad, and R. Agnès, 2000: Probability distributions for maximum wave and crest heights. Coast. Eng., 40, 329-360, https://doi.org/10.1016/S0378-3839(00) 00017-X.

Rademakers, P. J., 1993: Waverider-wavestaff comparison. Ocean Eng., 20, 187-193, https://doi.org/10.1016/0029-8018(93)90034-F.

Raghukumar, K., G. Chang, F. Spada, C. Jones, T. Janssen, and A. Gans, 2019: Performance characteristics of "Spotter," a newly developed real-time wave measurement buoy. J. Atmos.
Oceanic Technol., 36, 1127-1141, https://doi.org/10.1175/ JTECH-D-18-0151.1.

Rayleigh, L., 1915: Deep water waves, progressive or stationary, to the third order of approximation. Proc. Roy. Soc. London, 91A, 345-353, https://doi.org/10.1098/rspa.1915.0025.

Santamaria, F., G. Boffetta, M. M. Afonso, A. Mazzino, M. Onorato, and D. Pugliese, 2013: Stokes drift for inertial particles transported by water waves. Europhys. Lett., 102, 14003, https://doi.org/10.1209/0295-5075/102/14003.

Santo, H., P. H. Taylor, R. Eatock Taylor, and Y. S. Choo, 2013: Average properties of the largest waves in Hurricane Camille. J. Offshore Mech. Arct., 135, 011602, https://doi.org/10.1115/ 1.4006930.

Sharma, J. N., and R. G. Dean, 1981: Second-order directional seas and associated wave forces. Soc. Pet. Eng. J., 21, 129-140, https://doi.org/10.2118/8584-PA.

Srokosz, M. A., and M. S. Longuet-Higgins, 1986: On the skewness of sea-surface elevation. J. Fluid Mech., 164, 487-497, https:// doi.org/10.1017/S0022112086002653.

Stokes, G. G., 1847: On the theory of oscillatory waves. Trans. Cambridge Philos. Soc., 8, 441-455.

Tadjbakhsh, I., and J. B. Keller, 1960: Standing surface waves of finite amplitude. J. Fluid Mech., 8, 442-451, https://doi.org/ 10.1017/S0022112060000724.

Toffoli, A., M. Onorato, and J. Monbaliu, 2006: Wave statistics in unimodal and bimodal seas from a second-order model. Eur. J. Mech., 25B, 649-661, https://doi.org/10.1016/ j.euromechflu.2006.01.003.

_ J. Monbaliu, M. Onorato, A. R. Osborne, A. V. Babanin, and E. M. Bitner-Gregersen, 2007: Second-order theory and setup in surface gravity waves: a comparison with experimental data. J. Phys. Oceanogr., 37, 2726-2739, https://doi.org/10.1175/ 2007JPO3634.1.

van Essen, S., K. Ewans, and J. McConichie, 2018: Wave buoy performance in short and long waves, evaluated using tests on a hexapod. 37th Int. Conf. on Ocean, Offshore and Arctic Engineering, Madrid, Spain, American Society of Mechanical Engineers, V07BT06A001, https:/doi.org/10.1115/ OMAE2018-77092.

Walker, D. A. G., P. H. Taylor, and R. Eatock Taylor, 2004: The shape of large surface waves on the open sea and the Draupner New Year wave. Appl. Ocean Res., 26, 73-83, https://doi.org/ 10.1016/j.apor.2005.02.001.

Waseda, T., and Coauthors, 2014: Deep water observations of extreme waves with moored and free GPS buoys. Ocean Dyn., 64, 1269-1280, https://doi.org/10.1007/s10236-014-0751-4.

Whittaker, C. N., A. C. Raby, C. J. Fitzgerald, and P. H. Taylor, 2016: The average shape of large waves in the coastal zone. Coast. Eng., 114, 253-264, https://doi.org/10.1016/ j.coastaleng.2016.04.009. 